\title{
"Near-Perfect" Finite-Cardinality Generalized Space-Time Shift Keying
}

\author{
Chao Xu, Member, IEEE, Peichang Zhang, Member, IEEE, Rakshith Rajashekar, Senior Member, IEEE, Naoki \\ Ishikawa, Member, IEEE, Shinya Sugiura, Senior Member, IEEE, Zhaocheng Wang, Senior Member, IEEE, \\ and Lajos Hanzo* Fellow, IEEE
}

\begin{abstract}
Two decades of full-diversity high-rate MIMO research has created perfect Space-Time Block Codes (STBCs) including the Golden code. However, the major stumbling block of their wide-spread employment is their limited energy-efficiency. On one hand, the superposition of their signals results in a high Peak-to-Average Power Ratio (PAPR). On the other hand, the total number of equivalent Inter-Antenna Interference (IAI) contributions that the receiver has to deal with is increased to IAI $=M^{2}$ upon using $M$ Transmit Antennas (TAs), which is a substantial extra price compared to the IAI $=M$ of $\mathrm{V}$ BLAST. Against this background, we propose a new family of Finite-Cardinality Generalized Space-Time Shift Keying (FCGSTSK). More explicitly, the proposed FC-GSTSK is capable of outperforming both V-BLAST and STBC, which is the ultimate objective of full-diversity high-rate MIMO design. Furthermore, following the index modulation philosophy, the proposed FCGSTSK replaces the signal-additions by the data-carrying signalselection process. As a benefit, the FC-GSTSK substantially reduces the PAPR of signal transmission. As a further advantage, the equivalent IAI imposed on signal detection is reduced back to the same level as that of V-BLAST. Moreover, the proposed FCGSTSK is even capable of consistently outperforming the perfect STBCs in terms of its Peak Signal to Noise-power Ratio (PSNR) that takes into account the power consumption at the transmitter. As a further advance, the reduced-RF-chain based version of FC-GSTSK is also capable of outperforming both Generalized Spatial Modulation (GSM) and Space-Time Block Coded Spatial Modulation (STBC-SM) without increasing the PAPR and the equivalent IAI.
\end{abstract}

Index Terms-Space-time block code, perfect STBC, Golden code, generalized space-time shift keying, finite-cardinality, peak-to-average power ratio, inter-antenna interference, energyefficiency, bandwidth-efficiency, power-efficiency, full-RF-chain,

C. Xu, R. Rajashekar and L. Hanzo are with the School of Electronics and Computer Science, University of Southampton, Southampton SO17 1BJ, UK (e-mail: $\{$ cx1g08,rmr1u14,lh\}@ soton.ac.uk). P. Zhang is with College of Information Engineering, Shenzhen University, Shenzhen 518060, China (e-mail: pzhang@szu.edu.cn). N. Ishikawa is with the Graduate School of Information Sciences, Hiroshima City University, Ohzuka-higashi 7313194, Japan (e-mail: naoki@ishikawa.cc). S. Sugiura is with the Institute of Industrial Science, University of Tokyo, Meguro-ku, Tokyo 153-8505, Japan (e-mail: sugiura@ieee.org). Zhaocheng Wang is with Tsinghua University, Beijing, China (e-mail: zcwang@tsinghua.edu.cn).

L. Hanzo would like to acknowledge the financial support of the Engineering and Physical Sciences Research Council projects EP/Noo4558/1, EP/PO34284/1, COALESCE, of the Royal Societys Global Challenges Research Fund Grant, of the Royal Society Grant IF170002 as well as of the European Research Councils Advanced Fellow Grant QuantCom. The work of P. Zhang was supported in part by the Natural Science Foundation of China under Grant 61601304, in part by the Foundation of Shenzhen under Grant JCYJ20170302142545828, in part by the Foundation of Shenzhen University under Grant 2016057. The work of N. Ishikawa was supported in part by the Japan Society for the Promotion of Science KAKENHI under Grant 19K14987. The work of S. Sugiura was supported in part by the Japan Society for the Promotion of Science KAKENHI under Grants 17K18871 and $16 \mathrm{KK} 0120$.
reduced-RF-chain, index modulation, generalized spatial modulation.

\section{INTRODUCTION}

Following the recent digital and analog circuit breakthroughs in processing massive-bandwidth millimeter-wave signals, the next-generation mobile network is envisioned to achieve the ambitious goal of 1000 times higher capacity and 10-fold reduced roundtrip latency [1]. As a benefit of the substantial new available bandwidth, billions of powerhungry devices may be ubiquitously connected to the evolving Internet-of-Things (IoT), where the employment of energyefficient schemes associated with low-complexity transceivers is increasingly favorable. Against this backdrop, the invention of Spatial Modulation (SM) [2]-[4] harnessed the Index Modulation (IM) philosophy in the spatial domain, which inspired a paradigm shift from spectral-efficiency to energy-efficiency. More explicitly, the base-line SM activates a single one out of $M$ Transmit Antennas (TAs) in order to transmit a modulated $L$-PSK/QAM symbol. As a result, since only a single RF chain is required, the power consumption of the transmitter is substantially reduced [5]-[9]. Furthermore, the activation of a single TA effectively eliminates the Inter-Antenna Interference (IAI), which facilitates low-complexity single-stream-based detection [10], [11]. However, despite their attractive advantages in energy-efficiency, the IM design generally suffers from a reduced bandwidth-efficiency. For instance, the overall SM throughput is given by $R=\log _{2} M+\log _{2} L$, which is inferior to the V-BLAST throughput of $R=M \log _{2} L$ that grows linearly with $M$.

In the recent years, the IM philosophy has been intensively applied to a variety of scenarios [5]-[9] including Orthogonal Frequency-Division Multiplexing (OFDM) [12]-[14], FasterThan-Nyquist (FTN) [15]-[17] and differential Multiple-Input Multiple-Output (MIMO) systems [18]-[21], where the concept of energy-efficiency is not exclusively limited to the reduction of RF chains. In fact, the implementation of the single- and reduced-RF-chain designs still faces a number of practical challenges. First of all, the power amplifier of the single activated RF chain has to deliver $M$ times higher power than its counterparts operating in the full-RF mode. Secondly, the antenna switching time has to be substantially lower than the symbol period [22], and the time limit on pulse-shaping is also more strictly bounded, because the transmission of a whole symbol has to be completed over the activated symbol period [7]. Thirdly, the single- and reduced-RF designs are 
generally limited to the single-carrier systems, owing to the fact that the simultaneous activation of OFDM subcarriers would result in full-RF transmission.

Regardless of the number of activated RF chains, the actual signal constellation and the associated Peak-to-Average Power Ratio (PAPR) predetermine the power consumption of each RF chain. To this end, the diversity-aided IM schemes of Space-Time Shift Keying (STSK) and Generalized STSK (GSTSK) [23]-[27] that invoke arbitrary signals may not be deemed energy-efficient for the following reasons. First of all, the products of the communication industry typically use standardized PSK/QAM signals rather than arbitrary signals. The signal conversion from arbitrary signals to the FiniteCardinality (FC) fixed-point representation of PSK/QAM is subject to VLSI hardware constraints. Secondly, transmitting arbitrary signals undermine the low-PAPR advantage of singlecarrier systems. Thirdly, when a multi-carrier OFDM system is considered, typically the classic PSK/QAM constellations are used for frequency-domain modulation. Although the Inverse Fast Fourier Transform (IFFT) potentially increases the PAPR at the transmitter, a variety of practical techniques - including active constellation extension, selected mapping, tone injection and tone reservation [28]-[31] - are capable of reducing the time-domain OFDM signal's PAPR, but all of them operate based on the classic PSK/QAM input constellations before the IFFT.

Similar to STSK, the signal constellation typically becomes almost arbitrary when aiming for full-diversity high-rate MIMO design. Specifically, the family of perfect STBCs [32][35] including the Golden code [36] is capable of achieving unprecedented performance advantages over both V-BLAST and STBC relying on orthogonal [37], [38], on AmicableOrthogonal (AO) [39], [40] and on Quasi-Orthogonal (QO) [41], [42] designs. More explicitly, the perfect STBC partitions the $(M \times M)$ space-time signal matrix into $M$ layers, where each layer disperses a total number of $M$ modulated QAM/HEX [32] symbols. The beneficial cubic shaping property [32] ensures that the total power of each layer remains unchanged. Moreover, the state-of-the-art nonvanishing determinant property [32] further enhance the diversity gain. Despite the fact that the Golden code was included in the IEEE 802.16e-2005 WiMAX standard [43], the family of perfect STBCs is not sufficiently energy-efficient for next-generation MIMO systems. On one hand, owing to the superposition of their signals within each layer during the dispersion process, the associated constellation expansion and the increased PAPR impose extra constraints both on the Digital-to-Analog Converter (DAC) and on the power amplifier at the transmitter. On the other hand, in addition to the $M$ interfering elements within each layer, the space-time transmission of the $M$ layers also induces cross-layer IAIs. As a result, the total number of equivalent IAIs that the receiver has to deal with is increased to IAI $=M^{2}$, which is a substantial extra price compared to the conventional IAI $=M$ of V-BLAST. To alleviate the highPAPR, the so-called integer STBC arrangement was developed in [44], [45], where the transmitted signals are limited to an expanded $L^{2}$-QAM constellation. Moreover, in order to mitigate the excessive receiver complexity, the fast decoding algorithm and the so-called Silver code [43], [46], [47] endeavored to separate the in-phase and quadrature evaluations in the Sphere Decoder (SD). However, these solutions still suffer from both an increased PAPR compared to the single-stream $L$-QAM and from a performance loss over the optimal perfect STBC.

Against this background, we propose a new FC-GSTSK design, which is capable of outperforming both V-BLAST and $S T B C$ without increasing the PAPR, while the equivalent IAIs is reduced back to the moderate $I A I=M$ value of $V$-BLAST. Explicitly, the novel contributions of this paper are:

1) First of all, instead of using the irrational coefficients such as the Golden number of the Golden code, the dispersion elements of the new FC-GSTSK operate based on the $L_{D M}$-th root of unity $\left[w_{L_{D M}}=\exp \left(j \frac{2 \pi}{L_{D M}}\right)\right]$. Secondly, following the IM philosophy, the signalsuperposition form of $\left(\sum_{\bar{q}=1}^{\bar{Q}} s_{\bar{q}} w_{L_{D M}}^{\bar{q}}\right)$ is replaced by the signal-selection operation of $s^{l} w_{L_{D M}}^{\bar{q}}$, where both the modulation index $l$ and the activation index $\bar{q}$ carry source information. Thirdly, in order to maximize the diversity gains, the dispersion elements are appropriately rotated by the integers of $\left\{u_{m}\right\}_{m=1}^{M}$, as denoted by $\left\{w_{L_{D M}}^{u_{m} \bar{q}}\right\}_{m=1}^{M}$ in order to cater for the different MIMO setups. As a result, the benefits of the resultant full-RF FC-GSTSK are summarized in the following aspects:

(A) Energy-Efficiency: Thanks to the new dispersion elements and the IM design, the FC-GSTSK does not increase the PAPR of signal transmission. Moreover, the signal-selection process also eliminates the interference imposed on each layer. As a result, the total number of equivalent IAIs imposed on signal detection is reduced back to the $\mathrm{V}$ BLAST's IAI $=M$.

(B) Bandwidth-Efficiency: The FC-GSTSK throughput is given by $R=\log _{2} \bar{Q}+\log _{2} L$, which is higher than that of the family of STBCs [37]-[42]. We note that $\bar{Q}$ denotes the number of dispersion elements in each layer. Our analysis demonstrates that the bandwidth-efficiency of FC-GSTSK grows logarithmically with $\bar{Q}$ towards the full MIMO capacity, where increasing $\bar{Q}$ does not impose any extra hardware cost in terms of TAs, Receive Antennas (RAs) or RF chains.

(C) Power-Efficiency: As a full-diversity high-rate design, the FC-GSTSK massively outperforms STBCs [37]-[42], especially as $N$ and $R$ increase, where $N$ denotes the number of RAs. Moreover, the FC-GSTSK distinctly outperforms V-BLAST owing to its beneficial diversity gain. The improved power-efficiency of FC-GSTSK manifests itself in terms of its reduced power consumption required for achieving a certain target performance. Specifically, the FC-GSTSK is even capable of consistently outperforming the Golden code in terms of its Peak Signal to Noise-power Ratio (PSNR), which takes into account the practical power consumption of the transmitter.

(D) Deployability: The diversity gain optimization is 
conducted in an off-line fashion and all the FCGSTSK parameters are integers. Therefore, the transceiver is only required to store integer parameters instead of dispersion matrices or full signal matrices.

2) In this work, we are particularly interested in the FCGSTSK using the $L$-PSK modulation, which exhibits the perfect $0 \mathrm{~dB}$ PAPR. Nonetheless, in order to achieve a further improved performance at high throughputs, we conceive the Single-Element Amplitude Shift Keying (SE-ASK) as well as Multi-Element ASK (MEASK) for star QAM signalling. In particular, the MEASK arrangement achieves a substantially improved throughput at the cost of a slightly eroded diversity order. However, our simulation results confirm that the maximized diversity gain among a subset of FC-GSTSK codewords is sufficient to ensure the consistently superior performance of FC-GSTSK at high throughputs.

3) Moreover, in order to simplify the FC-GSTSK signal construction for using an increased number of $M$ TAs at high throughputs $R$, we additionally propose a DiversityRate (DR) tradeoff based arrangement. More explicitly, for an integer of $V=M / T$, where $T$ represents the number of time slots of a FC-GSTSK block, a $(T \times M)$ element FC-GSTSK is constructed by $V$ number of smaller $(T \times T)$ constituent FC-GSTSK. The resultant transmit diversity order is reduced to $[\min (M, T)=$ $T=M / V]$, but the throughput is improved to $[R=$ $\left.V\left(\log _{2} \bar{Q}+\log _{2} L\right)\right]$.

4) As a further advance, we develop the reduced-RF-chain version of FC-GSTSK, where a subset of $M_{A}$ out of $M$ TAs are activated. Our simulation results demonstrate that the reduced-RF FC-GSTSK is capable of outperforming both Generalized SM (GSM) and its diversityoriented counterpart of Space-Time Block Coded Spatial Modulation (STBC-SM) [48]-[51] without increasing the PAPR and the equivalent IAIs.

5) Finally, the proposed FC-GSTSK scheme is examined in conjunction with a low-complexity Binary Convolutional Coding (BCC) scheme, which is the channel coding arrangement of the IEEE $802.11 \mathrm{a} / \mathrm{n} / \mathrm{ac} / \mathrm{ah}$ standard family. The powerful analytical tool of EXtrinsic Information Transfer (EXIT) charts [52]-[55] is employed for analysing the convengence behaviour of iterative demapping and decoding. Our simulation results confirm the unequivocal performance advantages of the proposed FC-GSTSK scheme in the practical channel coded systems.

The new FC-GSTSK subsumes the single-RF STSK [21], which was proposed in the differential MIMO scenario and was shown to outperform Differential SM (DSM) [18], [19], [56], [57] and its diversity-oriented counterparts [20], [58][63]. Moving forward from [21], this work activates multiple RF chains and further improves the methodology of the diversity gain optimization, so that the new FC-GSTSK becomes capable of achieving the best MIMO performance.

The rest of this paper is organized as follows. The pre-

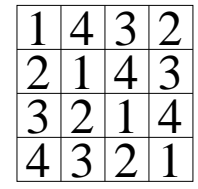

Fig. 1: Examples of the TAST layout [67] associated with $M=T=4$.

liminaries and system model are introduced in Sec. II. The signal construction of FC-GSTSK is proposed in Sec. III, while its further extensions to star QAM, DR and reduced$\mathrm{RF}$ arrangements are conceived in Sec. IV. Our performance results and conclusions are offered in Sec. V and Sec. VI, respectively.

The following notations are used throughout the paper. The operation $\otimes$ represents the Kronecker product, while $\operatorname{tr}(\cdot)$, $\operatorname{rank}(\cdot)$ and $\operatorname{det}(\cdot)$ take the trace, rank and determinant of a matrix, respectively. Moreover, for a generic MIMO scheme, the notations $M, N, T$ and $Q$ represent the numbers of TAs, RAs, transmission time slots and modulated symbols, respectively, while $L$ and $R$ represent the modulation alphabet size and throughput, respectively.

\section{Preliminaries and System Model}

First and foremost, it is important to portray our contributions in a historical perspective. Before the MIMO's space-time diversity era, the lattice-based multidimensional constellation design [64]-[66] endeavored to attain a beneficial diversity gain over the Single-Input Single-Output (SISO) systems. More explicitly, a sequence of $T$ dispersed symbols is given by:

$$
\overline{\mathbf{s}}=\frac{1}{\sqrt{T}} \mathbf{G}_{A} \mathbf{s} .
$$

The generator matrix $\mathbf{G}_{A}$ is of size $(T \times T)$, which is found to be a Vandermonde matrix $\mathbf{G}_{A}=\operatorname{VDM}_{T \times T}\left(g_{1}, \cdots, g_{T}\right)$ for the $L$-PSK modulation formulated as:

$$
\begin{aligned}
\mathbf{G}_{A}= & {\left[\begin{array}{lllll}
\bar{a}_{1,1} & \bar{a}_{1,2} & \bar{a}_{1,3} & \ldots & \bar{a}_{1, T} \\
\bar{a}_{2,1} & \bar{a}_{2,2} & \bar{a}_{2,3} & \ldots & \bar{a}_{2, T} \\
\vdots & \vdots & \vdots & \ddots & \vdots \\
\bar{a}_{T, 1} & \bar{a}_{T, 2} & \bar{a}_{T, 3} & \ldots & \bar{a}_{T, T}
\end{array}\right] } \\
= & {\left[\begin{array}{lllll}
1 & g_{1} & g_{1}^{2} & \ldots & g_{1}^{T-1} \\
1 & g_{2} & g_{2}^{2} & \ldots & g_{2}^{T-1} \\
\vdots & \vdots & \vdots & \ddots & \vdots \\
1 & g_{T} & g_{T}^{2} & \ldots & g_{T}^{T-1}
\end{array}\right] }
\end{aligned}
$$

The diversity is imposed by the dispersion operation of $\left(\bar{s}_{\bar{t}}=\sum_{t=1}^{T} \bar{a}_{\bar{t}, t} s_{t}=\sum_{t=1}^{T} g_{\bar{t}}^{t-1} s_{t}\right)$, where the dispersion elements $\left\{g_{\bar{t}}\right\}_{\bar{t}=1}^{T}$ are taken from a $4 T$-PSK constellation according to [64]. Therefore, instead of directly sending the modulated $L$-PSK symbols $\left\{s_{t}\right\}_{t=1}^{T}$ in $\mathbf{s}$ of (1), the correlated symbols $\left\{\bar{s}_{\bar{t}}\right\}_{\bar{t}=1}^{T}$ in $\overline{\mathbf{s}}$ are transmitted over the fading channel. As a result, the receiver may successfully recover $\overline{\mathbf{s}}=\left[\bar{s}_{1}, \cdots, \bar{s}_{T}\right]^{T}$, unless the whole sequence experiences a deep fade. There is no throughput loss for the SISO system given by $\left(R=\frac{T \log _{2} L}{T}=\log _{2} L\right)$. However, the PAPR of the transmitted symbols in $\overline{\mathbf{s}}$ of (1) is increased compared to the modulated symbols in $\mathbf{s}$ of (1), while the dispersion process also imposes $T$-element interferences among $\left\{\bar{s}_{\bar{t}}\right\}_{\bar{t}=1}^{T}$.

Accordingly, in order to achieve space-time diversity in MIMO systems, the Diagonal Algebraic Space-Time (DAST) design of [68] was proposed to transmit the dispersed sequence 
$\overline{\mathbf{s}}$ of (1) mapped to the diagonal of the MIMO's space-time signal matrix as:

$$
\begin{aligned}
& \mathbf{S}=\frac{1}{\sqrt{M}} \operatorname{diag}\left(\mathbf{G}_{A} \mathbf{s}\right) \\
& =\frac{1}{\sqrt{M}} \operatorname{diag}\left(\left[\sum_{m=1}^{M} g_{1}^{m-1} s_{m}, \sum_{m=1}^{M} g_{2}^{m-1} s_{m}, \cdots, \sum_{m=1}^{M} g_{M}^{m-1} s_{m}\right]\right) .
\end{aligned}
$$

The resultant throughput is also given by $\left(R=\frac{T \log _{2} L}{T}=\right.$ $\left.\log _{2} L\right)$. In order to regain the V-BLAST throughput of $R=M \log _{2} L$, the Threaded Algebraic Space-Time (TAST) arrangement [67] partitions a $(T \times M)$-element space-time signal-matrix into $M$ layers, where each layer transmits a dispersed sequence $\overline{\mathbf{s}}_{\tau}=\left\{\mathbf{G}_{A} \mathbf{s}_{\tau}\right\}_{\tau=1}^{M}$. The TAST layout is exemplified by Fig. 1. More explicitly, the signal matrix is constructed by [67]:

$$
\mathbf{S}=\frac{1}{M} \sum_{\tau=1}^{M} \phi_{r}^{\tau-1} \operatorname{diag}\left(\mathbf{G}_{A} \mathbf{S}_{\tau}\right) \mathbf{G}_{r}^{\tau-1} .
$$

The generator matrix $\mathbf{G}_{A}$ in (4) is still given by (2). The layer-switching phase rotations of $\left\{\phi_{r}^{\tau-1}=w_{L_{r}}^{\tau-1}\right\}_{\tau=1}^{M}$ in (4) are taken from a $L_{r}$-PSK constellation, which are invoked for the sake of retaining the full diversity order. Moreover, the layer-switching matrix $\mathbf{G}_{r}$ in (4) is given by:

$$
\mathbf{G}_{r}=\left[\begin{array}{ccccc}
0 & 0 & \cdots & 0 & 1 \\
1 & 0 & \cdots & 0 & 0 \\
0 & 1 & \cdots & 0 & 0 \\
\vdots & \vdots & \ddots & \vdots & \vdots \\
0 & 0 & \cdots & 1 & 0
\end{array}\right] .
$$

As a result, the TAST throughput is improved to $(R=$ $\left.\frac{M^{2} \log _{2} L}{T}=M \log _{2} L\right)$ for $M=T$. However, both the DAST and TAST designs suffer from the vanishing determinant problem, where the following diversity gain tends to zero as the throughput increases:

$$
\delta_{\min }=\min _{\forall \mathbf{S} \neq \mathbf{S}^{\prime}} \operatorname{det}\left[\left(\mathbf{S}-\mathbf{S}^{\prime}\right)\left(\mathbf{S}-\mathbf{S}^{\prime}\right)^{H}\right] .
$$

Following the success of the orthogonal STBC designs [37], [38], considerable research efforts have also been dedicated to finding the MIMO schemes that may achieve both fulldiversity and high-rate. Notably, it was demonstrated in [69] that a sufficient condition for achieving both the multiplexing and diversity gains quantified in [70] is to ensure a lower bound on the diversity gain of (6), which may be solved based on division algebra [69], [71], [72]. More explicitly, when the STBC signals are allowed to take values in an infinite constellation, the diversity gain of the infinite code is given by:

$$
\delta_{\text {inf }}=\min _{\forall \mathbf{S} \neq \mathbf{0}}|\operatorname{det}(\mathbf{S})|^{2},
$$

which is obtained by using $\left(\mathbf{S}^{\prime}=\mathbf{0}\right)$ and $(M=T)$ in (6). Naturally, the family of invertible matrices from division algebra guarantees $[\operatorname{det}(\mathbf{S}) \neq 0$ ] for $(7)$, which is sufficient for ensuring $\left(\delta_{\min } \neq 0\right)$ in (6). The division algebra designs generally use the following signal structure:

$$
\mathbf{S}=\frac{1}{\sqrt{M}} \sum_{\tau=1}^{M} \operatorname{diag}\left(\mathbf{G}_{A} \mathbf{s}_{\tau}\right) \mathbf{G}_{R}^{\tau-1},
$$

where the layer-switching phase $\phi_{R}$ is incorporated into the layer-switching matrix $\mathbf{G}_{R}$ as:

$$
\mathbf{G}_{R}=\left[\begin{array}{ccccc}
0 & 1 & 0 & \cdots & 0 \\
0 & 0 & 1 & \cdots & 0 \\
\vdots & \vdots & \vdots & \ddots & \vdots \\
0 & 0 & 0 & \cdots & 1 \\
\phi_{R} & 0 & 0 & \cdots & 0
\end{array}\right]
$$

Therefore, the optimization objective is to find the best $\mathbf{G}_{A}$ and $\phi_{R}$. Among all the devision algebra codes, the family of perfect STBCs [32]-[35] - including the Golden code [36] achieves the following outstanding properties:

(A) The cubic shaping property [32] requires $\mathbf{G}_{A}$ to be unitary, so that the overall power of each layer in (8) remains unchanged as $\left\{\left\|\mathbf{G}_{A} \mathbf{s}_{\tau}\right\|^{2}=\left\|\mathbf{s}_{\tau}\right\|^{2}\right\}_{\tau=1}^{T}$. Moreover, the average transmit power across the $M$ layers also remains the same, which requires $\phi_{R}$ to have unity power of $\left|\phi_{R}\right|^{2}=1$.

(B) The full V-BLAST throughput is retained for (8) as $\left(R=\frac{M^{2} \log _{2} L}{T}=M \log _{2} L\right)$, where a total number of $Q=M^{2}$ symbols are transmitted over $M=T$ times slots.

(C) The full STBC diversity order is achieved. Furthermore, the nondiminishing determinant property guarantees that without considering the power normalization, the diversity gain $\delta_{\text {inf }}$ of (7) is a non-zero constant $\delta_{c}$. More explicitly, when the modulated symbols $\left\{s_{q}\right\}_{q=1}^{Q}$ in $\left\{\mathbf{s}_{\tau}\right\}_{\tau=1}^{T}$ of (8) are given by Gaussian integers $\mathbb{Z}[j]$ or Eisenstein integers $\mathbb{Z}\left[w_{3}\right]$, where $w_{3}=\exp \left(j \frac{2 \pi}{3}\right)$ denotes the primitive third root of unity, the perfect STBC has ${ }^{1}$ :

$$
\delta_{\mathrm{inf}}=\frac{1}{\beta^{M}} \delta_{c} .
$$

The factor of $\frac{1}{\sqrt{\beta}}$ normalizes the average power of unbounded signal-set $\mathbb{Z}[j]$ or $\mathbb{Z}\left[w_{3}\right]$ to the unity-power cardinality- $L$ QAM/HEX constellations associated with $\left\{E\left(\left|s_{q}\right|^{2}\right)=1\right\}_{q=1}^{Q}$.

Example 1: The state-of-the-art Golden code [36] uses $\mathbf{G}_{A}=\frac{1}{\sqrt{5}}\left[\begin{array}{cc}\epsilon & \epsilon \theta \\ \bar{\epsilon} & \bar{\epsilon} \bar{\theta}\end{array}\right]$ and $\phi_{R}=j$, where the parameters are $\theta=\frac{1+\sqrt{5}}{2}, \epsilon=1+j-j \theta, \bar{\theta}=\frac{1-\sqrt{5}}{2}$ and $\bar{\epsilon}=$ $1+j-j \bar{\theta}$. As a result, the signal matrix of (8) becomes $\mathbf{S}=$ $\frac{1}{\sqrt{10}}\left[\begin{array}{cc}\epsilon\left(s_{1}+\theta s_{2}\right) & \epsilon\left(s_{3}+\theta s_{4}\right) \\ j \bar{\epsilon}\left(s_{3}+\bar{\theta} s_{4}\right) & \bar{\epsilon}\left(s_{1}+\bar{\theta} s_{2}\right)\end{array}\right]$, which uses $M=2$ TAs to convey $Q=4$ symbols $\left\{s_{q}\right\}_{q=1}^{4}$ over $T=2$ time slots. Given the relationships of $(\theta \bar{\theta}=-1),(\theta+\bar{\theta}=1)$ and $(\epsilon \bar{\epsilon}=2+j)$, we have $\operatorname{det}(\mathbf{S})=\frac{2+j}{10}\left[\left(s_{1}^{2}+s_{1} s_{2}-s_{2}^{2}\right)-j\left(s_{3}^{2}+s_{3} s_{4}-s_{4}^{2}\right)\right]$, which results $\operatorname{in}^{2} \delta_{\mathrm{inf}}=\frac{1}{20 \beta^{2}}$ for (7), where the minimum is given by $s_{1}=\frac{1}{\sqrt{\beta}}$ and $s_{2}=s_{3}=s_{4}=0$.

Although the cubic shaping property normalizes the layer power in the perfect STBC design, the PAPR of each transmitted symbol is inevitably increased due to the signals being multiplied and added together by $\mathbf{G}_{A} \mathbf{s}_{\tau}$ in (4). The associated constellation expansion is exemplied in Table I. As a result, it was shown in [44], [73] that the employment of realistic finite-precision DACs may impose a severe performance loss on the Golden code. Furthermore, Table I also shows that the perfect STBCs impose an equivalent IAI $=M^{2}$ on the signal detection at the receiver. More explicitly, the transmitted

${ }^{1} \mathrm{We}$ note that owing to the power normalization for ensuring $E\left[\operatorname{tr}\left(\mathbf{S S}^{H}\right)\right]=T$ in the MIMO systems design, the diversity gain of the perfect code still declines as the throughput increases.

${ }^{2}$ Once again, owing to the normalization factors of $\frac{1}{\sqrt{\beta}}$ for $\left\{E\left(\left|s_{q}\right|^{2}\right)=\right.$ $1\}_{q=1}^{Q}$ and $\frac{1}{\sqrt{M}}$ in (8) for $E\left[\operatorname{tr}\left(\mathbf{S S}^{H}\right)\right]=T$, the diversity gain derived in this work is smaller than the one originally claimed in [36]. 
TABLE I: Constellation diagrams and energy-efficiency measures of Golden code [36] and perfect STBCs [32]-[35].

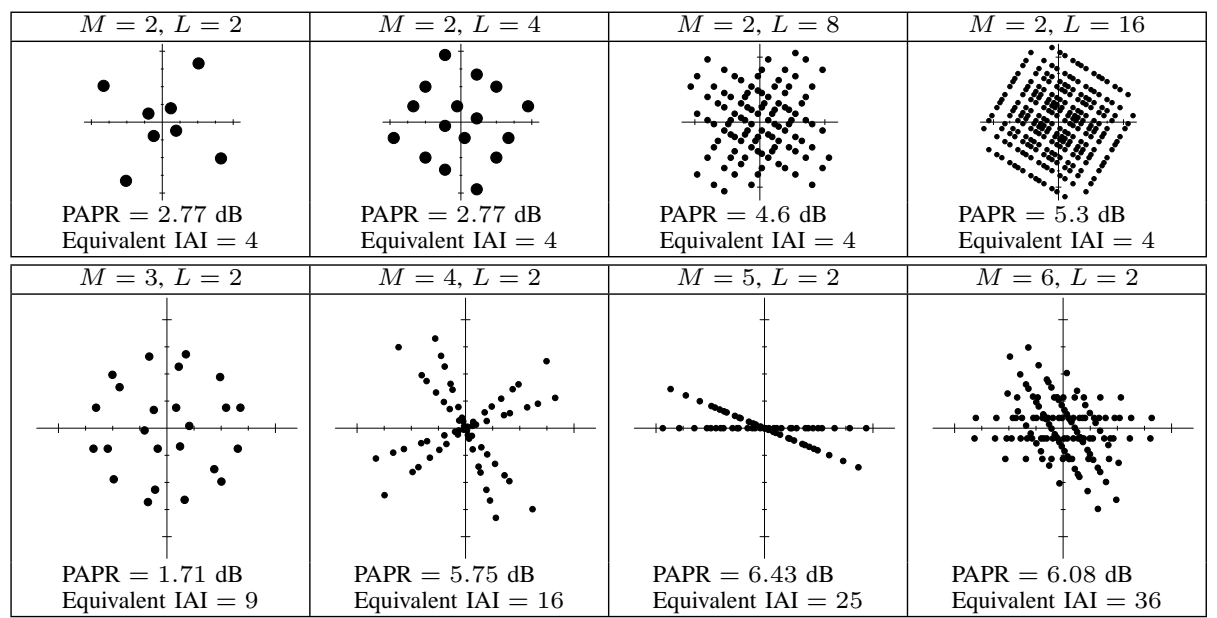

signals of (8) may be expressed in the form of the Linear Dispersion Code (LDC) [74] as:

$$
\mathbf{S}=\sum_{\tau=1}^{M} \sum_{m=1}^{M} s_{\tau, m} \mathbf{A}_{\tau, m}=\sum_{q=1}^{Q} \bar{s}_{q} \overline{\mathbf{A}}_{q}
$$

where we have $\bar{s}_{q}=s_{\tau, m}$ and $\overline{\mathbf{A}}_{q}=\mathbf{A}_{\tau, m}=\frac{1}{\sqrt{M}} \operatorname{diag}\left\{\mathbf{G}_{A}(\right.$ : $, m)\} \mathbf{G}_{R}^{\tau-1}$, while $\mathbf{G}_{A}(:, m)$ denotes the $m$-th column in $\mathbf{G}_{A}$. The relationship between indices is given by $[q=m+(\tau-$ 1) $M]$. Moreover, the signal received by the $N$ RAs over $T$ times slots may be modeled as:

$$
\mathbf{Y}=\mathbf{S H}+\mathbf{V}
$$

where the $(T \times M)$-element matrix $\mathbf{S}$ and the $(T \times N)$ element matrix $\mathbf{Y}$ represent the input and output signals of the MIMO channels, while the $(M \times N)$-element $\mathbf{H}$ and $(T \times N)$-element $\mathbf{V}$ model the Rayleigh fading channels and the AWGN, respectively. As a result, vectorizing $\mathbf{Y}$ of (12) leads to the following LDC signal model:

$$
\overline{\mathbf{Y}}=\overline{\mathbf{S}} \overline{\mathbf{H}}+\overline{\mathbf{V}}
$$

where we have the $(1 \times T N)$-element $\overline{\mathbf{Y}}=\operatorname{rvec}(\mathbf{Y})$ and $\overline{\mathbf{V}}=$ $\operatorname{rvec}(\mathbf{V})$. The equivalent $(1 \times Q)$-element transmitted signal vector $\overline{\mathbf{S}}$ is given by:

$$
\overline{\mathbf{S}}=[\underbrace{s_{1,1} \cdots s_{1, M}}_{\tau=1} \underbrace{s_{2,1} \cdots s_{2, M}}_{\tau=2} \cdots \underbrace{s_{M, 1} \cdots s_{M, M}}_{\tau=M}] .
$$

The $(Q \times T N)$-element channel matrix is $\overline{\mathbf{H}}=\bar{\chi}\left(\mathbf{I}_{T} \otimes\right.$ $\mathbf{H})$, where we have the $(Q \times T M)$-element $\bar{\chi}=$ $\left[\operatorname{rvec}\left(\overline{\mathbf{A}}_{1}\right)^{T}, \cdots, \operatorname{rvec}\left(\overline{\mathbf{A}}_{Q}\right)^{T}\right]^{T}$. As a result, the signal detection of the perfect STBC is equivalent to that of a V-BLAST scheme associated with $Q=M^{2}$ TAs and $T N$ RAs. Hence the total number of equivalent IAIs that the receiver has to mitigate is increased to IAI $=M^{2}$.

\section{IndEX MOdulation AsSisted FULl-Diversity High-RATE SignAl CONSTRUCTION}

In this section, we propose the novel FC-GSTSK in Sec. III-A, where the schematic descriptions are summarized in Sec. III-B. The bespoke diversity gain maximization is presented in Sec. III-C, and our analysis of the bandwidthand power-efficiency is offered in Sec. III-D.

\section{A. The Energy-Efficient Finite-Cardinality Design}

Following the IM philosophy, all the signal additions in (4) and (8) may be replaced by data-carrying signal-selection. First of all, we propose to rewrite the $\tau$-th layer in (4) and (8) as:

$$
\operatorname{diag}\left(\mathbf{G}_{A} \mathbf{s}_{\tau}\right)=s^{l_{\tau}}\left[\begin{array}{llll}
w_{L_{D M}}^{(\bar{q}-1) u_{1}} & & & \\
& w_{L_{D M}}^{(\bar{q}-1) u_{2}} & & \\
& & \ddots & \\
& & & w_{L_{D M}}^{(\bar{q}-1) u_{M}}
\end{array}\right],
$$

where the generator matrix $\mathbf{G}_{A}$ is revised to be an $(M \times \bar{Q})$ element Vandermonde matrix as:

$$
\begin{aligned}
\mathbf{G}_{A}= & \operatorname{VDM}_{M \times \bar{Q}}\left(w_{L_{D M}}^{u_{1}}, \cdots, w_{L_{D M}}^{u_{M}}\right) \\
& =\left[\begin{array}{ccccc}
1 & w_{L_{D M}}^{u_{1}} & w_{L_{D M}}^{2 u_{1}} & \ldots & w_{L_{D M}}^{(\bar{Q}-1) u_{1}} \\
1 & w_{L_{D M}}^{u_{2}} & w_{L_{D M}}^{2 u_{2}} & \ldots & w_{L_{D M}}^{(\bar{Q}-1) u_{2}} \\
\vdots & \vdots & \vdots & \ddots & \vdots \\
1 & w_{L_{D M}}^{u_{M}} & w_{L_{D M}}^{2 u_{M}} & \ldots & w_{L_{D M}}^{(\bar{Q}-1) u_{M}}
\end{array}\right],
\end{aligned}
$$

while the $(\bar{Q} \times 1)$-element IM vector $\mathbf{s}_{\tau}$ is given by:

$$
\mathbf{s}_{\tau}=\left[\begin{array}{lllllll}
0 & \cdots & 0 & s^{l_{\tau}} & 0 & \cdots & 0
\end{array}\right]^{T} .
$$

We note that the choice of $\bar{Q}$ becomes flexible, which is independent of the hardware parameter of the number of TAs, RAs and RF chains. As a result, the activation index $\bar{q}_{\tau}$ and the modulation index $l_{\tau}$ carry $\log _{2} \bar{Q}$ and $\log _{2} \bar{L}$ information bits, respectively. Following this, we propose to construct the FC-GSTSK based on the TAST signal structure of (4) as:

$$
\mathbf{S}=\frac{1}{\sqrt{M}} \sum_{\tau=1}^{M} \phi_{r}^{\tau-1} \operatorname{diag}\left(\mathbf{G}_{A} \mathbf{S}_{\tau}\right) \mathbf{G}_{r}^{\tau-1}
$$

where the layer-switching phase is given by $\phi_{r}=w_{L_{r}}$, while the layer-switching matrix $\mathbf{G}_{r}$ is given by (5). The integer rotation-parameters $\mathbf{u}=\left[\begin{array}{lll}u_{1} & \cdots & u_{m}\end{array}\right]$ are introduced in order to maximize the diversity gain, which will be detailed in Sec. III-C. In summary, the overall FC-GSTSK throughput is given by $\left[R=\frac{M\left(\log _{2} \bar{Q}+\log _{2} L\right)}{T}=\log _{2} \bar{Q}+\log _{2} L\right]$ for $M=T$, which is higher than that of STBCs from orthogonal [37], [38], AO [39], [40] and QO [41], [42] designs.

Furthermore, the LDC form [74] for the FC-GSTSK of (18) may be expressed as:

$$
\mathbf{S}=\sum_{\tau=1}^{M} s^{l_{\tau}} \mathbf{A}_{\tau, \bar{q}_{\tau}}=\sum_{q=1}^{Q} \bar{s}_{q} \overline{\mathbf{A}}_{q}
$$


where the symbols $\left\{\bar{s}_{q}\right\}_{q=1}^{Q}$ are given by:

$$
\bar{s}_{q}= \begin{cases}s^{l_{\tau}}, & \text { for } q=\bar{q}_{\tau}+(\tau-1) \bar{Q} \\ 0, & \text { for all the other } q\end{cases}
$$

while the dispersion matrices are expressed by:

$$
\begin{aligned}
& \overline{\mathbf{A}}_{q}=\mathbf{A}_{\tau, \bar{q}} \\
& \quad=\frac{1}{\sqrt{M}} \phi_{r}^{\tau-1} \operatorname{diag}\left(\left[w_{L_{D M}}^{(\bar{q}-1) u_{1}}, w_{L_{D M}}^{(\bar{q}-1) u_{2}}, \cdots, w_{L_{D M}}^{(\bar{q}-1) u_{M}}\right]\right) \mathbf{G}_{r}^{\tau-1} .
\end{aligned}
$$

The relationship between the indices is given by $[q=$ $\bar{q}+(\tau-1) \bar{Q}]$. It is readily seen that the PAPR of $s^{l_{\tau}}$ remains unchanged, since the $m$-th signal on the $\tau$-th layer in the FC-GSTSK signal matrix of (18) is given by $\frac{1}{\sqrt{M}} s^{l_{\tau}} w_{L_{D M}}^{\left(\bar{q}_{\tau}-1\right) u_{m}} w_{L_{r}}^{\tau-1}$. Furthermore, the equivalent LDC received signal model is also given by (13), where the equivalent $(1 \times Q)$-element transmitted signal vector $\overline{\mathbf{S}}$ is now given by:

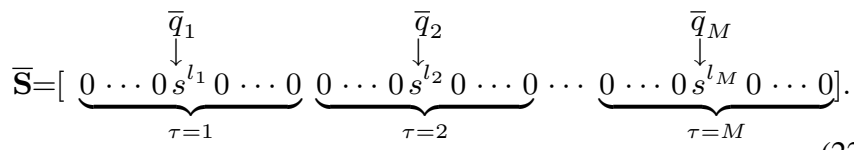

As a result, the FC-GSTSK signal detection is equivalent to that of a GSM scheme associated with $Q=M \bar{Q}$ TAs and $M$ activated RF chains. Thanks to the IM design, the equivalent IAI is reduced to $\mathrm{IAI}=M$ in (22).

Remark 1 (Parameters): The FC-GSTSK scheme of (18) is unambiguously specified by the integer parameters of $(M$, $\left.N, T, \bar{Q}, L, L_{D M}, L_{r}, \mathbf{u}\right)$, where we have $M=T$ and $Q=\bar{Q} M$. Given a MIMO setup of $M$ TAs and $N$ RAs, the diversity gain maximization process detailed in Sec. III-C is responsible for finding the best parameters of $\left(L_{D M}, L_{r}, \mathbf{u}\right)$ that determine $\mathbf{G}_{A}$ and $\phi_{r}$ for each specific throughput target of $\left[R=\log _{2} \bar{Q}+\log _{2} L\right]$.

Remark 2 ( $\boldsymbol{R F}$ chains): Owing to the simultaneous activation of all layers in (18), the default mode of FC-GSTSK is the full-RF transmission with IAI $=M$. Nonetheless, if " $\sum_{\tau=1}^{M}$ " in (18) is replaced by " $\sum_{\tau=1}^{M_{A}}$ ", a subset of $M_{A}$ out of $M$ layers is activated, which results in a reduced number of $M_{A}$ activated RFs associated with IAI $=M_{A}$. For the special case of $M_{A}=1$, the FC-GSTSK of (18) becomes the single-RF and IAI-free STSK design of [21].

Remark 3 (Complexity): According to [26], the ML detection complexity of FC-GSTSK that activates $M$ out of $Q$ dispersion matrices is in proportional to the full ML complexity within a factor of $M / Q$ for the LDCs of (4) and (8) in Sec. II. When the suboptimal GSM detectors of [49], [50] are considered, a MMSE detector or a SD of the equivalent $M$ IAI V-BLAST may be invoked $\bar{Q}^{M}$ times for all the activation combinations in (22). In this work, the ML detectors of [26], [27] are assumed in order to verify the performance analysis of FC-GSTSK.

\section{B. Schematic Description}

The schematics of Goden code and FC-GSTSK are explicitly visualized in Fig. 2. As demonstrated by Fig. 2(a), the diversity advantage of Goden code [36] is achieved at the substantial extra cost for the transceiver to deal with the increased PAPR and IAI, which constitutes the major stumbling block of the practical deployment of perfect STBCs

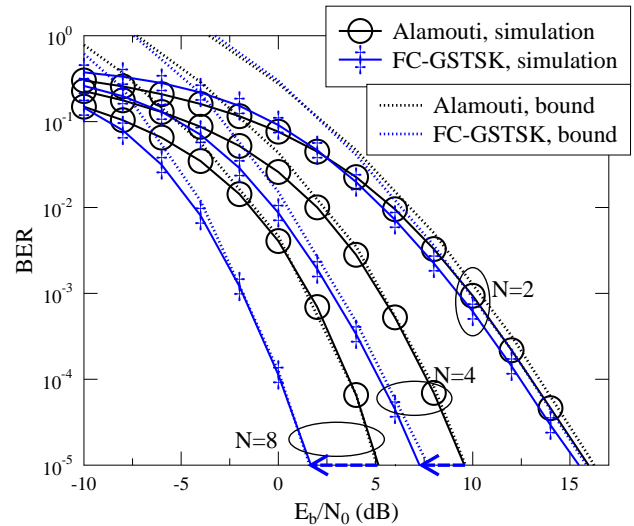

Fig. 3: BER comparison between Alamouti's G2 STBC and the new FC-GSTSK, when we have $(M=T=2), N=\{2,4,8\}$ and $R=3.0$. The parameters can be found in Sec. V.

[32]-[36]. By contrast, the proposed FC-GSTSK conceived based on the TAST and IM philosophies is capable of offering the following exclusive advantages:

(A) The IM design eliminates the signal superpositions in TAST, as demonstrated by Fig. 2(b). As a result, when specifically considering $L$-PSK signalling for $s^{l_{\tau}}$, the perfect $0 \mathrm{~dB}$ PAPR is retained for all of the FC-GSTSK signals of $\left\{s^{l_{\tau}} w_{L_{D M}}^{\left(\bar{q}_{\tau}-1\right) u_{m}} w_{L_{r}}^{\tau-1}\right\}_{\tau=1}^{M}$.

(B) The IM design eliminates the intra-layer IAI, as demonstrated by Fig. 2(b). As a result, the total IAI of the FC-GSTSK is reduced back to the same level as the V-BLAST's IAI $=M$.

(C) The FC-GSTSK throughput is given by $R=\log _{2} \bar{Q}+$ $\log _{2} L$, where increasing $\bar{Q}$ does not impose extra hardware cost in terms of more TAs, RAs or RF chains. The FC-GSTSK capacity will be further analysed in Sec. III-D.

(D) The FC-GSTSK retains the non-diminishing high diversity gain by appropriately adjusting the integer parameters of $\left(L_{D M}, L_{r},\left\{u_{m}\right\}_{m=1}^{M}\right)$ in order to cater for the different MIMO setups, which will be further elaborated on in Sec. III-C.

\section{Diversity Gain Maximization}

The averaged BER of a generic MIMO scheme is given by: [55], [75]

$$
\bar{P}_{e, b i t} \leq \mathrm{E}\left[\sum_{i=0}^{I-1} \sum_{i^{\prime}=0}^{I-1} \frac{d_{H}\left(i, i^{\prime}\right)}{I \log _{2} I} p\left(\mathbf{S}^{i} \rightarrow \mathbf{S}^{i^{\prime}}\right)\right],
$$

where $d_{H}\left(i, i^{\prime}\right)$ refers to the Hamming distance between the bit-mappings of $\mathbf{S}^{i}$ and $\mathbf{S}^{i^{\prime}}$. The Pairwise Error Probability (PEP) is upper bounded by: [55], [75]

$$
p\left(\mathbf{S}^{i} \rightarrow \mathbf{S}^{i^{\prime}}\right) \leq \mathrm{E}\left\{Q\left[\frac{\sqrt{\left\|\left(\mathbf{S}^{i}-\mathbf{S}^{i^{\prime}}\right) \mathbf{H}\right\|^{2}}}{2 N_{0}}\right]\right\}
$$

where $Q(\cdot)$ represents the integral form of the Q-function. The BERs of the Alamouti's G2 STBC and the proposed FCGSTSK are exemplied in Fig. 3, which verifies the advantages of FC-GSTSK in terms of both simulation and the theoretical bound of (23). Furthermore, in order to formulate the Objective 


\begin{tabular}{|c|c|c|c|c|c|c|c|c|c|c|}
\hline \multicolumn{10}{|c|}{ Golden Code } & 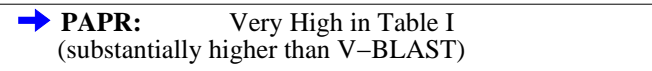 \\
\hline $\mathbf{S}=s_{1}$ & $\frac{\epsilon}{\sqrt{10}}$ & $\begin{array}{l}0 \\
\frac{\bar{\epsilon}}{\sqrt{10}}\end{array}$ & $+s_{2}$ & {$\left[\begin{array}{c}\frac{\epsilon \theta}{\sqrt{10}} \\
0\end{array}\right.$} & $\begin{array}{c}0 \\
\frac{\bar{\epsilon} \bar{\theta}}{\sqrt{10}}\end{array}$ & $+\underbrace{s_{3}}$ & {$\left[\begin{array}{cc}0 & \frac{\epsilon}{\sqrt{10}} \\
j \frac{\bar{\epsilon}}{\sqrt{10}} & 0\end{array}\right.$} & $+s_{4}$ & {$\left[\begin{array}{cc}0 & \frac{\epsilon \theta}{\sqrt{10}} \\
j \frac{\bar{\epsilon} \bar{\theta}}{\sqrt{10}} & 0\end{array}\right]$} & $\rightarrow$ Total IAI: IAI $=M^{2}=4$ \\
\hline & & & aver & & & -Layer & Intra & aye & & $\begin{array}{ll}\rightarrow \text { Throughput: } & R=M \log _{2} L \text { (same as V-BLAST) } \\
\rightarrow \text { Diversity: } & \text { Non-Diminishing High Diversity Gain }\end{array}$ \\
\hline
\end{tabular}

(a) Golden Code
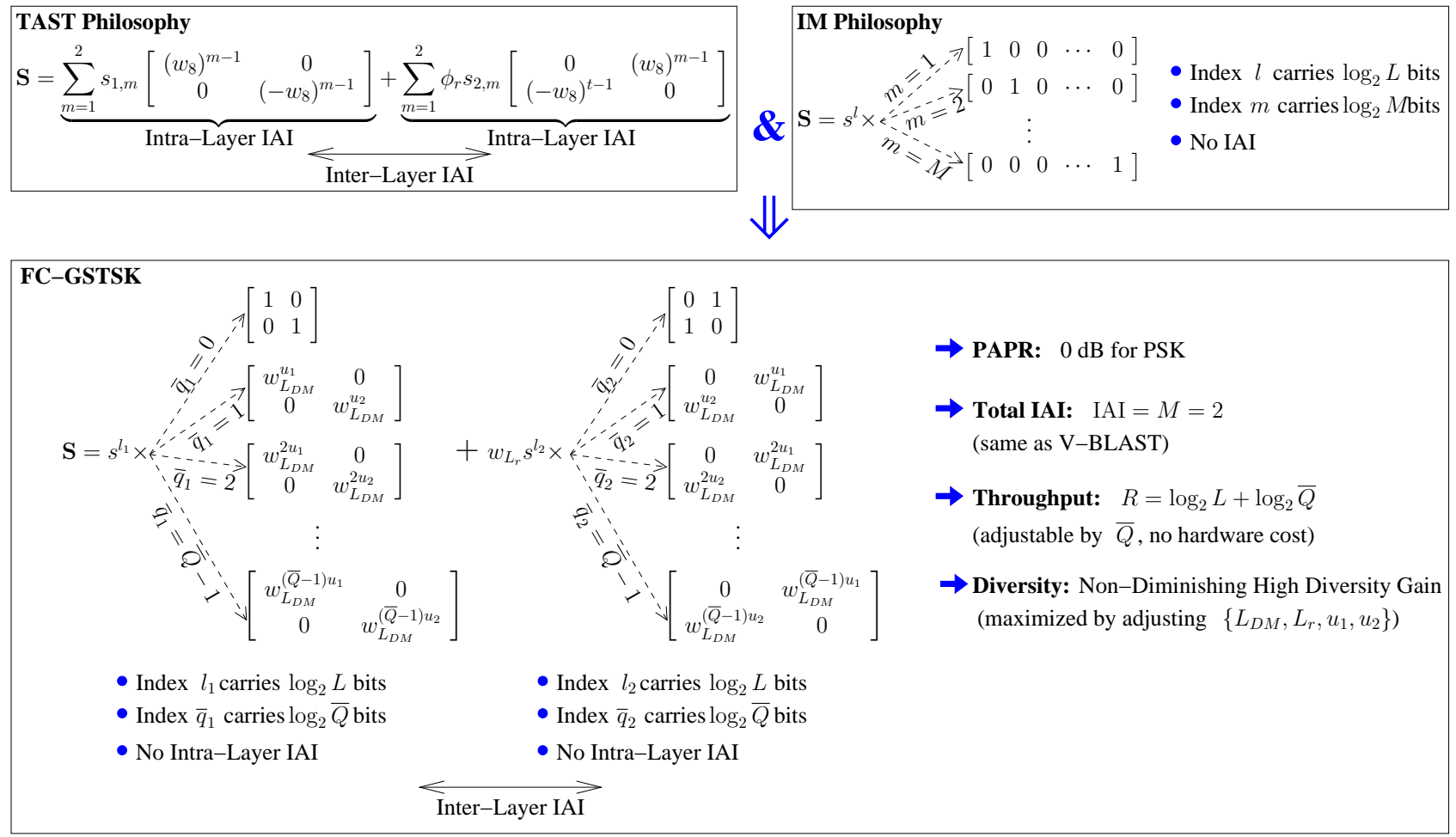

(b) FC-GSTSK

Fig. 2: Schematic descriptions of Golden code [36] seen in Example 1 and the proposed FC-GSTSK $(M=T=2)$.

Functions (OFs) of diversity gains, the PEP of (24) is extended as:

$$
p\left(\mathbf{S}^{i} \rightarrow \mathbf{S}^{i^{\prime}}\right) \leq\left(\frac{1}{4 N_{0}}\right)^{-N \operatorname{rank}(\boldsymbol{\Delta})}\left[\prod_{m=1}^{\operatorname{rank}(\boldsymbol{\Delta})} \lambda_{m}\right]^{-N}
$$

where $\left\{\lambda_{m}\right\}_{m=1}^{M}$ are eigenvalues of $\left[\boldsymbol{\Delta}=\left(\mathbf{S}^{i}-\mathbf{S}^{i^{\prime}}\right)^{H}\left(\mathbf{S}^{i}-\mathbf{S}^{i^{\prime}}\right)\right]$. Assuming the full $\operatorname{rank}[\operatorname{rank}(\boldsymbol{\Delta})=M=T]$, the PEP may be further rewritten as: [76]

$$
p\left(\mathbf{S}^{i} \rightarrow \mathbf{S}^{i^{\prime}}\right) \leq\left[1+\sum_{m=1}^{M}\left(\frac{1}{4 N_{0}}\right)^{m} \Xi_{m}\right]^{-N},
$$

where $\left(\Xi_{m}=\sum_{1 \leq i_{1}<i_{2}<\cdots<i_{m} \leq M} \prod_{k=1}^{m} \lambda_{i_{k}}\right)$ has the special cases of $\left(\Xi_{M}=\prod_{m=1}^{M} \lambda_{m}\right)$ and $\left(\Xi_{1}=\sum_{m=1}^{M} \lambda_{m}\right)$. Owing to the associated coefficients of $\left(\frac{1}{4 N_{0}}\right)^{M}$ and $\left(\frac{1}{4 N_{0}}\right)^{1}$ for $\Xi_{M}$ and $\Xi_{1}$ in (26), the determinant term $\left[\operatorname{det}(\boldsymbol{\Delta})=\prod_{m=1}^{M} \lambda_{m}\right]$ and the trace term $\left[\operatorname{tr}(\boldsymbol{\Delta})=\sum_{m=1}^{M} \lambda_{m}\right]$ dominate the PEP in the highand low-SNR regions, respectively. Accordingly, the trend of PEP may be characterized by the diversity product and by the diversity sum as [21], [76]:

$$
\begin{aligned}
& \Lambda_{p}=\frac{1}{2} \min _{\forall i \neq i^{\prime}} \operatorname{det}(\boldsymbol{\Delta})^{\frac{1}{2 T}}, \\
& \Lambda_{s}=\frac{1}{2 \sqrt{T}} \min _{\forall i \neq i^{\prime}} \operatorname{tr}(\boldsymbol{\Delta})^{\frac{1}{2}} .
\end{aligned}
$$

Moreover, according to the average BER expression of (23), the average diversity product and average diversity sum are expressed as [21]:

$$
\begin{aligned}
& \bar{\Lambda}_{p}=\frac{1}{2}\left[\frac{2}{\log _{2} I+1} \sum_{\forall i} \sum_{\forall i^{\prime} \neq i} \frac{d_{H}\left(i, i^{\prime}\right)}{I \log _{2} I \operatorname{det}(\boldsymbol{\Delta})}\right]^{-\frac{1}{2 M}}, \\
& \bar{\Lambda}_{s}=\frac{1}{2 \sqrt{M}}\left[\frac{2}{\log _{2} I+1} \sum_{\forall i} \sum_{\forall i^{\prime} \neq i} \frac{d_{H}\left(i, i^{\prime}\right)}{I \log _{2} I \operatorname{tr}(\boldsymbol{\Delta})}\right]^{-\frac{1}{2}} .
\end{aligned}
$$

The four metrics of $\Lambda_{p}, \Lambda_{s}, \bar{\Lambda}_{p}$ and $\bar{\Lambda}_{s}$ in (27) and (28) are normalized to be within $[0,1]$. Fig. 4(a) demonstrates that a higher $\Lambda_{p}$ or $\Lambda_{s}$ leads to better performance for smaller $N=$ $\{1,2\}$ or larger $N=\{8,16\}$, respectively. Moreover, Fig. 4(b) further confirms that when $\Lambda_{p}$ and $\Lambda_{s}$ are fixed, the higher values of $\bar{\Lambda}_{p}$ and $\bar{\Lambda}_{s}$ also lead to better performance.

Unfortunately, it is impossible to maximize all the four OFs of $\left\{\Lambda_{p}, \Lambda_{s}, \bar{\Lambda}_{p}, \bar{\Lambda}_{s}\right\}$ at the same time. Therefore, it is important to determine their priorities according to the MIMO setup. There are a total number of $4 !=24$ possible arrangements of priorities, and our analysis is characterized by the following linear regression equation:

$$
\mathrm{SNR}_{t}=\kappa_{0}+\kappa_{1} \Lambda_{p}+\kappa_{2} \Lambda_{s}+\kappa_{3} \bar{\Lambda}_{p}+\kappa_{4} \bar{\Lambda}_{s},
$$

where for each construction of $\left\{\mathbf{S}^{i}\right\}_{i=0}^{I-1}$ according to (18), the simulation result of $\mathrm{SNR}_{t}$ is recorded at the target $\mathrm{BER}=10^{-4}$, while the associated $\left\{\Lambda_{p}, \Lambda_{s}, \bar{\Lambda}_{p}, \bar{\Lambda}_{s}\right\}$ are 


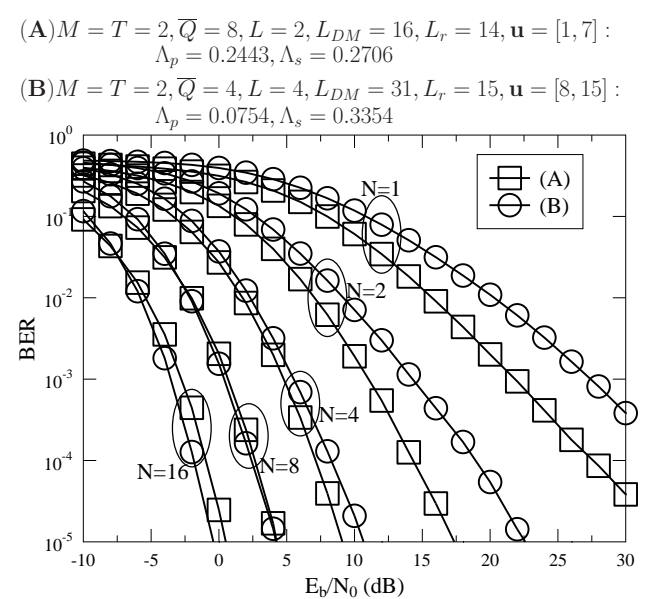

(a) Comparison between $\Lambda_{p}$ and $\Lambda_{s}$

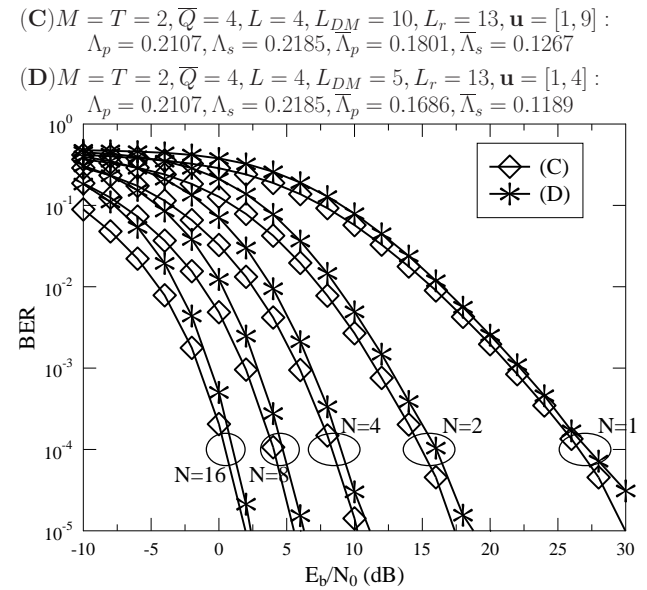

(b) Comparison for $\bar{\Lambda}_{p}$ and $\bar{\Lambda}_{s}$

Fig. 4: Performance comparison between four FC-GSTSK $(M=T=$ $2, R=4)$ associated with different parameters.

evaluated based on (27) and (28). The regression coefficients $\left\{\kappa_{0}, \cdots, \kappa_{4}\right\}$ can be automatically determined by machine learning in Excel, and the descending priorities are assigned to the four OFs according to their descending coefficient values.

More explicitly, the pseudocode for the diversity gain maximization of the FC-GSTSK is provided in Table II, which summarizes four proto-type arrangements that are often encountered in our intensive simulations. The first two arrangements in Table II often produce beneficial FC-GSTSK solutions for small $N=\{1,2\}$, while the latter two arrangements often lead to good performance for $N>2$. Following this, the rest of the pseudocode in Table II aims for finding the best integer parameters $\left(1<L_{D M}, L_{r} \leq L T \bar{Q}\right)$ and $\left(1 \leq\left\{u_{m}\right\}_{m=1}^{M}<L_{D M}\right)$ that maximize the four OFs $\left\{\Lambda_{1}, \cdots, \Lambda_{4}\right\}$ in descending order of priorities. We note that the values of $\left\{u_{m}\right\}_{m=1}^{M}$ has to be relatively prime to $L_{D M}$, as represented by $\left\{\operatorname{gcd}\left(u_{m}, L_{D M}\right)=1\right\}_{m=1}^{M}$ in Table II. Otherwise, there exist $\left[u_{m}(q-1) \bmod L_{D M}=0\right]$ values that result in $\left(\Lambda_{p}=0\right)$.

Example 2: For the case of $M=T=2$ and $R=3.0$, the best FC-GSTSK we have found has the parameters of $(\bar{Q}=$ $\left.4, L=2, L_{D M}=8, L_{r}=14, \mathbf{u}=[1,3]\right)$. This is obtained by performing full-search over all the possible combinations of $\left(1<L_{D M} \leq 16\right),\left(1<L_{r} \leq 16\right)$ and $\left\{\operatorname{gcd}\left(u_{m}, L_{D M}\right)=\right.$ $1\}_{m=1}^{M}$. The signal constructions of (16), (21) and (22) are
TABLE II: Pseudocode for the diversity gain maximization of the FC-GSTSK.

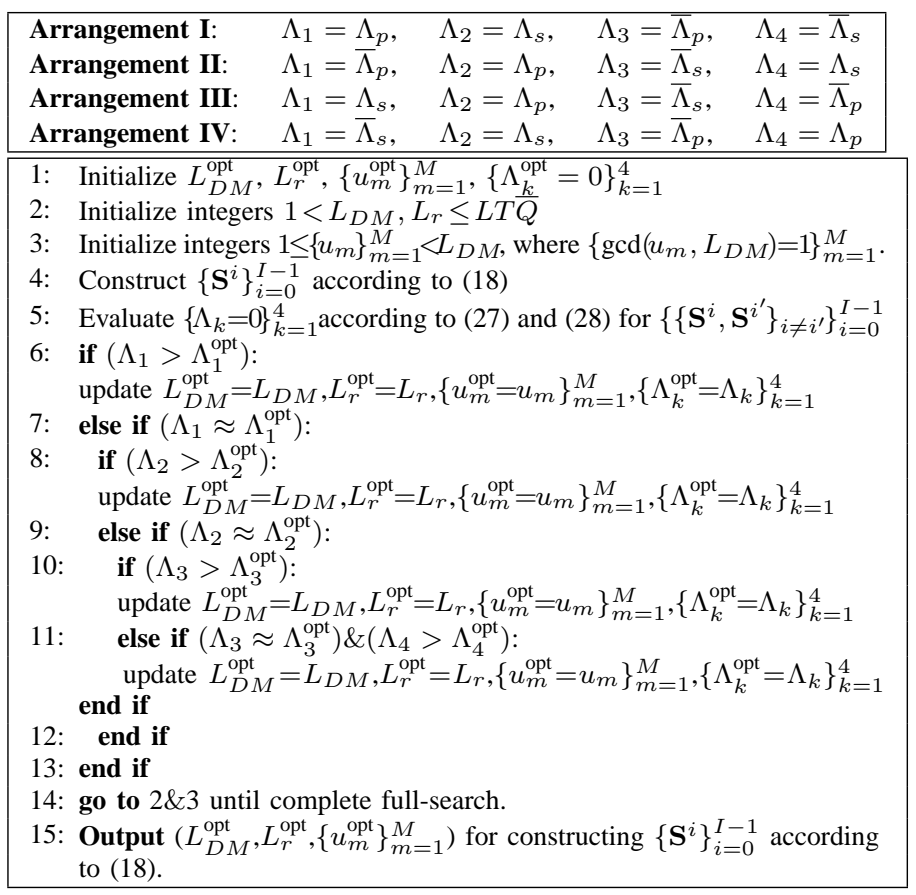

TABLE III: Signal constructions of (16), (21) and (22) for the FCGSTSK of Example 2: using $\left(\bar{Q}=4, L=2, L_{D M}=8, L_{r}=\right.$ $14, \mathbf{u}=[1,3])$.

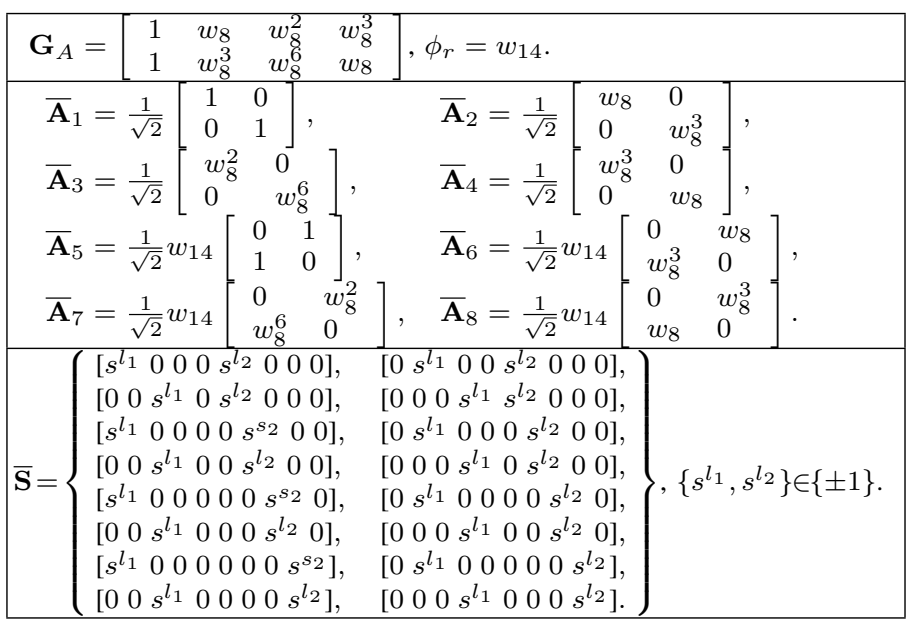

presented in Table III. The resultant signal matrices $\mathbf{S}$ of (18) have $\left(\Lambda_{p}=0.3917\right)$ and $\left(\Lambda_{s}=0.5\right)$, which are substantially higher than $\left(\Lambda_{p}=0.2706\right)$ and $\left(\Lambda_{s}=0.2706\right)$ of Alamouti's STBC [37] using 8PSK. Hence it is expected that FC-GSTSK is capable of substantially outperforming Alamouti's STBC using $M=T=2$ and $R=3.0$ (see Fig. 8b).

Remark 4 (High-throughput construction): At high throughtputs, the full-search of Table II may become infeasible. Alternatively, one may invoke Table II for the DAST signal model of $\mathbf{S}=\operatorname{diag}\left(\mathbf{G}_{A} \mathbf{S}\right)$ in order to obtain $L_{D M}$ and $\left\{u_{m}\right\}_{m=1}^{M}$ as the first step. For example, for the case of $M=T=2$ and $R=8.0$, there are a total number of $\left(I=2^{R T}=65536\right)$ combinations for the FC-GSTSK signal matrices, but this is reduced to only $\left(I=2^{R T / 2}=256\right)$ for the DAST. Upon obtaining $L_{D M}$ and $\left\{u_{m}\right\}_{m=1}^{M}$, Table II may be invoked again just for $L_{r}$. 


\section{Analysis on Bandwidth- and Power-Efficiency}

First of all, let us examine the bandwidth-efficiency with the aid of the Continuous-input Continuous-output Memoryless Channel (CCMC) capacity. More explicitly, the CCMC capacity of V-BLAST evaluated based on $[p(\mathbf{Y} \mid \mathbf{S})=$ $\left.\frac{1}{\left(\pi N_{0}\right)^{N}} \exp \left(-\frac{\|\mathbf{Y}-\mathbf{S H}\|^{2}}{N_{0}}\right)\right]$ is given by:

$$
\mathcal{C}_{\mathrm{MIMO}}^{\mathrm{CCMC}}(\mathrm{SNR})=\max _{p(\mathbf{S})} \mathcal{I}(\mathbf{S} ; \mathbf{Y})=\mathrm{E}\left[\log _{2} \operatorname{det}\left(\mathbf{I}_{N}+\frac{\eta}{M} \mathbf{H}^{H} \mathbf{H}\right)\right],
$$

where $\left(\mathrm{SNR}=10 \log _{10} \eta \mathrm{dB}\right)$, while the mutual information $\mathcal{I}(\mathbf{S} ; \mathbf{Y})$ is maximized for a Gaussian input signal having $\left[\mathrm{E}\left(\mathbf{S}^{H} \mathbf{S}\right)=\frac{1}{M} \mathbf{I}_{M}\right]$.

By contrast, for the orthogonal STBCs [37]-[40], the signal construction may be generalized as $\left[\mathbf{S}=\sqrt{P_{t}} \sum_{q=1}^{Q}\left(\mathbf{D}_{q}^{+} s_{q}+\right.\right.$ $\left.\left.\mathbf{D}_{q}^{-} s_{q}^{*}\right)\right]$, where the power normalization factor $P_{t}$ ensures $E\left[\operatorname{tr}\left(\mathbf{S S}^{H}\right)\right]=T$, while $\left\{\mathbf{D}_{q}^{+}, \mathbf{D}_{q}^{-}\right\}_{q=1}^{Q}$ are dispersion matrices from orthogonal design. Owing to the orthogonality, the conditional probability $p(\mathbf{Y} \mid \mathbf{S})$ may be decoupled as $[p(\mathbf{Y} \mid \mathbf{S})=$ $\left.\vartheta \prod_{q=1}^{Q} p\left(\bar{z}_{q} \mid s_{q}\right)\right]$, where we have $\left\{\bar{z}_{q}=\frac{M Q \sqrt{P_{t}}}{T\|\mathbf{H}\|^{2}} \operatorname{tr}\left[\mathbf{Y}^{H} \mathbf{D}_{q}^{-} \mathbf{H}+\right.\right.$ $\left.\left.\mathbf{H}^{H}\left(\mathbf{D}_{q}^{+}\right)^{H} \mathbf{Y}\right]\right\}, \quad\left[\vartheta=\frac{\left(\pi \bar{N}_{0}\right)^{Q}}{\left(\pi N_{0}\right)^{N T}} \exp \left(-\frac{\varrho}{N_{0}}\right)\right], \quad\left[\varrho=\|\mathbf{Y}\|^{2}-\right.$ $\left.\sum_{q=1}^{Q} \frac{T\|\mathbf{H}\|^{2}}{M Q}\left|\bar{z}_{q}\right|^{2}\right]$ and $\left[p\left(\bar{z}_{q} \mid s_{q}\right)=\frac{1}{\pi \bar{N}_{0}} \exp \left(-\frac{\left|\bar{z}_{q}-s_{q}\right|^{2}}{\bar{N}}\right)\right]$, while the equivalent noise power is given by $\left(\bar{N}_{0}=\frac{M Q}{T\|\mathbf{H}\|^{2}} N_{0}\right)$. As a result, the CCMC capacity of STBCs from orthogonal design marks a loss in bandwidth-efficiency as [55], [77]:

$$
\begin{aligned}
& \mathcal{C}_{S T B C}^{C C M C}(S N R)=\max _{\left\{p\left(s_{q}\right)\right\}_{q=1}^{Q}} \frac{1}{T} \sum_{q=1}^{Q} \mathcal{I}\left(s_{q} ; \bar{z}_{q}\right) \\
& \quad=\frac{Q}{T} \mathrm{E}\left[\log _{2}\left(1+\frac{T\|\mathbf{H}\|^{2}}{M Q} \eta\right)\right], \\
& =\frac{Q}{T} C_{M I M O}^{C C M C}\left(M N, 1, \frac{N T}{Q} \eta\right) \leq C_{M I M O}^{C C M C}(M, N, \eta),
\end{aligned}
$$

which indicates that $\mathcal{C}_{\mathrm{STBC}}^{\mathrm{CCMC}}(\mathrm{SNR})$ is equivalent to a fraction $\frac{Q}{T}$ of the full MIMO capacity of (30) with $M N$ TAs, 1 RA and $\left(\mathrm{SNR}=10 \log _{10} \frac{N T}{Q} \eta \mathrm{dB}\right)$. Therefore, the orthogonal STBC only becomes capacity-optimal for the special case of Alamouti's $(M=2)$ scheme using $(N=1)$.

Moreover, for the generic LDC signal model of (13), the CCMC capacity evaluated based on $[p(\overline{\mathbf{Y}} \mid \overline{\mathbf{S}})=$ $\left.\frac{1}{\left(\pi N_{0}\right)^{T N}} \exp \left(-\frac{\|\overline{\mathbf{Y}}-\overline{\mathbf{S H}}\|^{2}}{N_{0}}\right)\right]$ is given by [55], [74]:

$$
\begin{aligned}
& \mathcal{C}_{\mathrm{LDC}}^{\mathrm{CCMC}}(\mathrm{SNR})=\max _{p(\overline{\mathbf{s}})} \mathcal{I}(\overline{\mathbf{s}} ; \overline{\mathbf{Y}}) \\
& \quad=\mathrm{E}\left\{\log _{2} \operatorname{det}\left[\mathbf{I}_{N T}+\eta\left(\mathbf{I}_{T} \otimes \mathbf{H}\right)^{H} \bar{\chi}^{H} \bar{\chi}\left(\mathbf{I}_{T} \otimes \mathbf{H}\right)\right]\right\} .
\end{aligned}
$$

In particular, the family of the capacity-optimal LDCs [55], [74] including the Golden code [36] has $\bar{\chi}^{H} \bar{\chi}=\frac{1}{M} \mathbf{I}_{T M}$, which results in the full MIMO capacity of $\left[\mathcal{C}_{\mathrm{LDC}}^{\mathrm{CCMC}}(\mathrm{SNR})=\right.$ $\left.\mathcal{C}_{\text {MIMO }}^{\text {CCMC }}(\mathrm{SNR})\right]$.

For the proposed FC-GSTSK, both the classic modulated symbols $\left\{s^{l_{\tau}}\right\}_{\tau=1}^{M}$ and the IM activation indices $\left\{\bar{q}_{\tau}\right\}_{\tau=1}^{M}$ in (22) carry input information, yielding:

$$
\mathcal{C}_{\text {FC-GSTSK }}^{\text {CCMC }}(\mathrm{SNR})=\mathcal{C}_{\text {FC-GSTSK }}^{\text {CCMC-MOD }}(\mathrm{SNR})+\mathcal{C}_{\text {FC-GSTSK }}^{\text {CCMC-IM }}(\mathrm{SNR}) .
$$

The first part in (33) is evaluated based on $[p(\overline{\mathbf{Y}} \mid \overline{\mathbf{S}})=$ $\left.\frac{1}{\left(\pi N_{0}\right)^{T N}} \exp \left(-\frac{\|\overline{\mathbf{Y}}-\overline{\mathbf{S H}}\|^{2}}{N_{0}}\right)\right]$ as:

$$
\begin{aligned}
& \mathcal{C}_{\mathrm{FC}-\mathrm{GSTSK}}^{\mathrm{CCMC-MOD}}(\mathrm{SNR})=\max _{\left\{p\left(s^{l} \tau\right)\right\}_{\tau=1}^{M}} \frac{1}{T} \mathcal{I}\left(\left\{s^{l_{\tau}}\right\}_{\tau=1}^{M} ; \overline{\mathbf{Y}} \mid\left\{\bar{q}_{\tau}\right\}_{\tau=1}^{M}\right)
\end{aligned}
$$

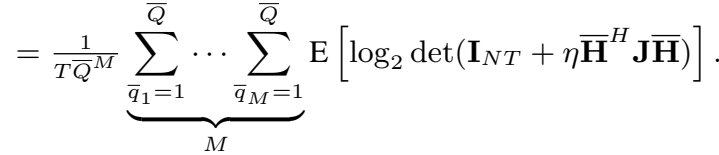

The $(Q \times Q)$-element $\left[\mathbf{J}=\mathrm{E}\left(\overline{\mathbf{S}}^{H} \overline{\mathbf{S}}\right)=\operatorname{diag}\left(\mathbf{J}_{1}, \mathbf{J}_{2}, \cdots, \mathbf{J}_{M}\right)\right]$ is conditioned on the IM activation indices $\left\{\bar{q}_{\tau}\right\}_{\tau=1}^{M}$, where the constituent $(\bar{Q} \times \bar{Q})$-element $\left\{\mathbf{J}_{\tau}\right\}_{\tau=1}^{M}$ has all-zero elements except for a single non-zero element on the $\bar{q}_{\tau}$-th row and $\bar{q}_{\tau}$ column as:

$$
\mathbf{J}_{\tau}=\left[\begin{array}{ccccccc}
0 & \cdots & 0 & 0 & 0 & \cdots & 0 \\
\vdots & \ddots & \vdots & \vdots & \vdots & . & \vdots \\
0 & \cdots & 0 & 0 & 0 & \cdots & 0 \\
0 & \cdots & 0 & 1 & 0 & \cdots & 0 \\
0 & \cdots & 0 & 0 & 0 & \cdots & 0 \\
\vdots & . & \vdots & \vdots & \vdots & \ddots & \vdots \\
0 & \cdots & 0 & 0 & 0 & \cdots & 0
\end{array}\right] .
$$

The mutual information in (34) is maximized for the "Gaussianized" signals of $\left\{s^{l_{\tau}}\right\}_{\tau=1}^{M}$. By contrast, the IM activation indices $\left\{\bar{q}_{\tau}\right\}_{\tau=1}^{M}$ are always discrete-valued. More explicitly, the second part in (33) is evaluated based on $\left[p\left(\overline{\mathbf{Y}} \mid\left\{\bar{q}_{\tau}\right\}_{\tau=1}^{M}\right)=\right.$ $\left.\frac{1}{\operatorname{det}\left(\pi \mathbf{R}_{Y Y}\right)} \exp \left(-\overline{\mathbf{Y}} \mathbf{R}_{Y Y}^{-1} \overline{\mathbf{Y}}\right)\right]$ relying on $\left[\mathbf{R}_{Y Y}=\mathrm{E}\left(\overline{\mathbf{Y}}^{H} \overline{\mathbf{Y}}\right)=\right.$ $\left.\overline{\mathbf{H}}^{H} \mathbf{J} \overline{\mathbf{H}}+N_{0} \mathbf{I}_{N T}\right]$ as (36), where the mutual information is maximized for the equal-prob source of $\left\{p\left(\bar{q}_{\tau}\right)=1 / \bar{Q}\right\}_{\tau=1}^{M}$. As a result, the bandwidth-efficiency of FC-GSTSK increases logarithmically with $\bar{Q}$, which is evidenced by Fig. 5(a). Furthermore, Fig. 5(a) also demonstrates that as $\bar{Q}$ grows, the CCMC capacity of FC-GSTSK improves further beyond STBC towards the full MIMO capacity. Once again, we note that increasing the parameter $\bar{Q}$ in the FC-GSTSK signal model of (18) does not impose extra hardware cost in terms of TAs, RAs or RF chains.

The CCMC capacities of Fig. 5(a) are unbounded upon increasing the SNR. However, in reality, the power-efficiency at a specific target rate is more relevant to the overall system performance. The encountered Discrete-input Continuousoutput Memoryless Channel (DCMC) capacity is evaluated based on $\left[p(\mathbf{Y} \mid \mathbf{S})=\frac{1}{\left(\pi N_{0}\right)^{N T}} \exp \left(-\frac{\|\mathbf{Y}-\mathbf{S H}\|^{2}}{N_{0}}\right)\right]$ as:

$$
\begin{aligned}
\mathcal{C}^{\text {DCMC }}(\mathrm{SNR}) & =\max _{p(\mathbf{S})} \frac{1}{T} \sum_{\forall \mathbf{S}} \int p(\mathbf{Y} \mid \mathbf{S}) p(\mathbf{S}) \log _{2} \frac{p(\mathbf{Y} \mid \mathbf{S})}{p(\mathbf{Y})} d \mathbf{Y} \\
& =\frac{1}{I T} \sum_{i=0}^{I-1} \mathrm{E}\left[\log _{2} \frac{I \exp \left(-\frac{\left\|\mathbf{Y}-\mathbf{S}^{i} \mathbf{H}\right\|^{2}}{N_{0}}\right)}{\sum_{i^{\prime}=0}^{I-1} \exp \left(-\frac{\left\|\mathbf{Y}-\mathbf{S}^{i^{\prime} \mathbf{H}}\right\|^{2}}{N_{0}}\right)}\right],
\end{aligned}
$$

which is maximized for the equal-prob source of $\left\{p\left(\mathbf{S}^{i}\right)=\right.$ $1 / I\}_{i=0}^{I-1}$.

As a result, it is demonstrated by Fig. 5(b) that the proposed FC-GSTSK is capable of achieving better DCMC capacities than V-BLAST and STBC, where the SNRs required for the FC-GSTSK to achieve the target rates of $R=\{2.0,3.0,4.0\}$ are lower, hence the better powerefficiency. Moreover, it also appears that as the throughput increases to $R=4.0$, the Golden code starts to perform the best in Fig. 5(b). However, when the practical power consumption at the transmitter is taken into account by the PSNR of (PSNR $\left.=10 \log _{10} \frac{\max |s|}{N_{0}} \mathrm{~dB}\right)$, the power-efficiency of FC-GSTSK is substantially better than that of the Golden code, as distinctively marked in Fig. 5(c). Our more detailed performance comparisons will be presented in Sec. V.

\section{EXTENSIONS ON Signal CONSTRUCTION}

In this section, we proceed to extend the FC-GSTSK signal construction for a wider range of MIMO scenarios. First of all, the ASK design for star QAM signalling is presented in 


$$
\begin{aligned}
\mathcal{C}_{\mathrm{FC}-\mathrm{GSTSK}}^{\mathrm{CCMCIM}}(\mathrm{SNR}) & =\max _{\left\{p\left(\overline{\bar{q}}_{\tau}\right)\right\}_{\tau=1}^{M}} \frac{1}{T} \mathcal{I}\left(\left\{\bar{q}_{\tau}\right\}_{\tau=1}^{M} ; \overline{\mathbf{Y}}\right)=\max _{\left\{p\left(\bar{q}_{\tau}\right)\right\}_{\tau=1}^{M}} \frac{1}{T} \underbrace{\bar{Q}}_{\bar{q}_{1}=1} \cdots \sum_{\bar{q}_{M}=1}^{\bar{Q}} \int p\left(\overline{\mathbf{Y}} \mid\left\{\bar{q}_{\tau}\right\}_{\tau=1}^{M}\right) \prod_{\tau=1}^{M} p\left(\bar{q}_{\tau}\right) \log _{2} \frac{p\left(\overline{\mathbf{Y}} \mid\left\{\bar{q}_{\tau}\right\}_{\tau=1}^{M}\right)}{p(\overline{\mathbf{Y}})} d \overline{\mathbf{Y}} \\
& =\frac{1}{T \bar{Q}^{M}} \underbrace{\sum_{\bar{q}_{1}=1}{ }^{\bar{Q}} \cdots \sum_{\bar{q}_{M}=1}^{\bar{Q}} \mathrm{E}}_{M}\left[\log _{2} \frac{\bar{Q}^{M} p\left(\overline{\mathbf{Y}} \mid\left\{\bar{q}_{\tau}\right\}_{\tau=1}^{M}\right)}{\sum_{\bar{q}_{1}^{\prime}=1}^{M} \cdots \sum_{\bar{q}_{M}^{\prime}=1}^{\bar{Q}} p\left(\overline{\mathbf{Y}} \mid\left\{\bar{q}_{\tau}^{\prime}\right\}_{\tau=1}^{M}\right)}\right]
\end{aligned}
$$

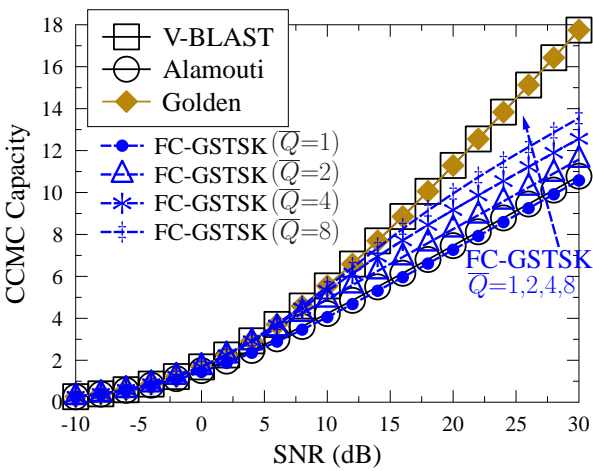

(a) Bandwidth-Efficiency: CCMC

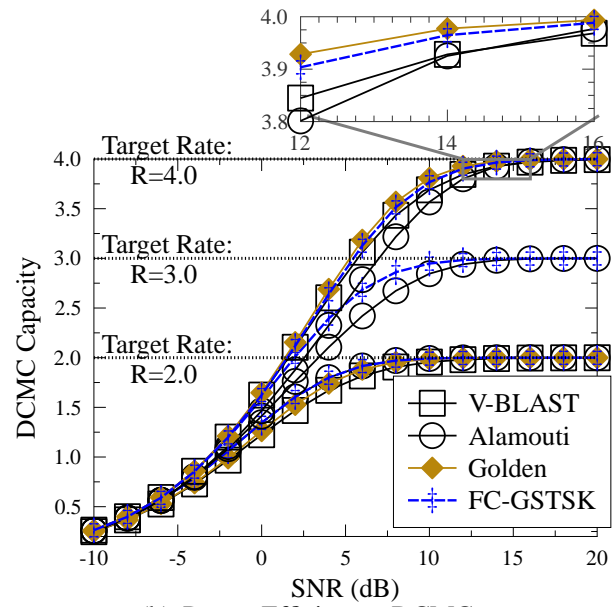

(b) Power-Efficiency: DCMC

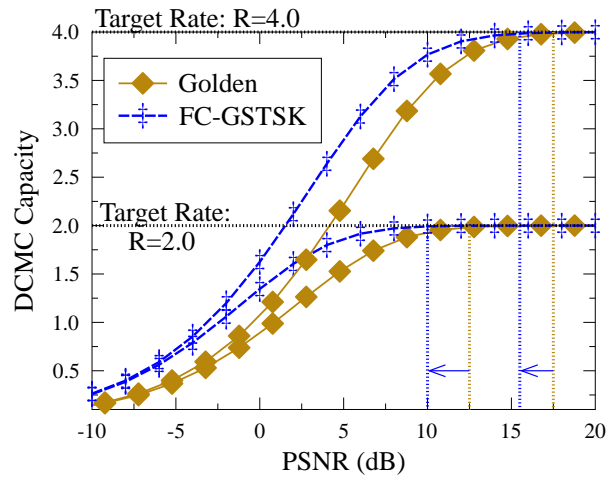

(c) Power-Efficiency: DCMC (PSNR)

Fig. 5: CCMC and DCMC capacity comparison between V-BLAST, Alamouti's STBC, Golden code and FC-GSTSK associated with $M=2$ and $N=2$. The parameters for FC-GSTSK are given by Table VI in Sec. V.

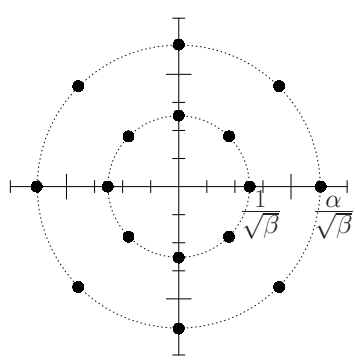

(a) $L_{A}=2, L=8$

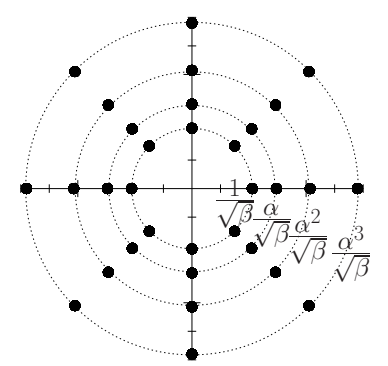

(b) $L_{A}=4, L=8$

Fig. 6: Constellation diagrams for star QAM associated with $L_{A}=\{2,4\}$ ASK-amplitudes and $L=\{8,16\}$ PSK-phases.

Sec. IV-A. Secondly, the DR arrangement for higher number of TAs is conceived in Sec. IV-B. Lastly, the reduced-RF version of FC-GSTSK is proposed in Sec. IV-C.

\section{A. Amplitude Shift Keying (ASK)}

As shown in Sec. III-A, the FC-GSTSK does not increase the signal transmission PAPR. Specifically, when the square and star QAM are used for the modulated symbols in $\mathbf{s}_{\tau}$ of (18), the constellation of the FC-GSTSK's transmitted signals in $\mathbf{S}$ of (18) is always limited to star QAM. Therefore, in this section, we explicitly conceive the design guidelines for using SE-ASK and ME-ASK for star QAM signalling, where the ASK-amplitudes are modulated independently of the phase matrix, so that the notations of Sec. III remain the same.

For the SE-ASK design, in contrast to the single-RF schemes [20], [21], [56], [57] that invoke a single ASK for the whole signal matrix, the FC-GSTSK modulates a $L_{A}$-level ASK symbol $\gamma_{\tau}$ for each layer as:

$$
\mathbf{S}=\frac{1}{\sqrt{M}} \sum_{\tau=1}^{M} \phi_{r}^{\tau-1} \gamma_{\tau} \operatorname{diag}\left(\mathbf{G}_{A} \mathbf{S}_{\tau}\right) \mathbf{G}_{r}^{\tau-1},
$$

TABLE IV: Parameters $\left(\bar{Q}, L, L_{D M}, L_{r}, \mathbf{u}\right)$ of FC-GSTSK (PSK) and parameters $\left(\bar{Q}, L, L_{D M}, L_{r}, \mathbf{u}, L_{A}, \alpha\right)$ of FC-GSTSK (PSK,SE/ME-ASK) used in Fig. 7.

\begin{tabular}{|l|l|}
\hline \multirow{2}{*}{$R=6.0$} & PSK: $(4,16,64,54,[7,57])$ \\
& SE-ASK: $(4,8,97,82,[5,80], 2,2.0)$ \\
& ME-ASK: $(4,4,10,14,[1,9], 2,2.0)$ \\
\hline \multirow{2}{*}{$R=8.0$} & PSK:(16, 16, 256,7, $[1,161])$ \\
& SE-ASK: $(4,16,64,15,[1,49], 4,1.4)$ \\
& ME-ASK: $(4,16,64,23,[1,15], 2,2.0)$ \\
\hline
\end{tabular}

where we have $\left\{\left\{\gamma_{\tau}=\frac{\alpha^{a_{\tau}}}{\sqrt{\beta}}\right\}_{a_{\tau}=1}^{L_{A}}\right\}_{\tau=1}^{M}$, while $\alpha$ and $\beta=$ $\frac{\sum_{\mu=0}^{L_{A}-1} \alpha^{2 \mu}}{L_{A}}$ respectively represent the ring ratio and the associated normalization factor that ensures $\left[E\left(\gamma_{\tau}^{2}\right)=1\right]$. The resultant star QAM constellations are exemplied in Fig. 7. We note that the advantageous choices of ring ratios in Rayleigh fading scenarios are $\alpha=2.0$ for $L_{A}=2$ and $\alpha=1.4$ for $L_{A}=4$ [78]-[81]. In summary, the overall throughput is improved to $\left[R=\log _{2} \bar{Q}+\log _{2} L+\log _{2} L_{A}\right]$.

As a further advance, the ME-ASK design [21], [56], [57] is revised to modulate a total of $M$ different ASK symbols in each FC-GSTSK layer as:

$$
\mathbf{S}=\frac{1}{\sqrt{M}} \sum_{\tau=1}^{M} \phi_{r}^{\tau-1} \boldsymbol{\Lambda}_{\tau} \operatorname{diag}\left(\mathbf{G}_{A} \mathbf{s}_{\tau}\right) \mathbf{G}_{r}^{\tau-1}
$$

where we have $\boldsymbol{\Lambda}_{\tau}=\operatorname{diag}\left(\left[\gamma_{\tau, 1}, \cdots, \gamma_{\tau, M}\right]\right)$. The further improved throughput is given by $\left[R=\log _{2} \bar{Q}+\log _{2} L+\right.$ $\left.M \log _{2} L_{A}\right]$. The ME-ASK design no longer retains the full diversity. For example, the two signal matrices $\mathbf{S}^{i}$ and $\mathbf{S}^{i^{\prime}}$ of (39) associated with a single different data-carrying ASKamplitude $\left(\gamma_{1,1} \neq \gamma_{1,1}^{\prime}\right)$ but the same remaining source bits have rank-1 $\boldsymbol{\Delta}$ for the PEP of (25). However, $\mathbf{G}_{A}$ and $\phi_{r}$ optimized from Table II for the phase matrices still offer a performance advantage for FC-GSTSK(PSK,ME-ASK) over V-BLAST, as evidenced by Fig. 7. This is due to the fact that 


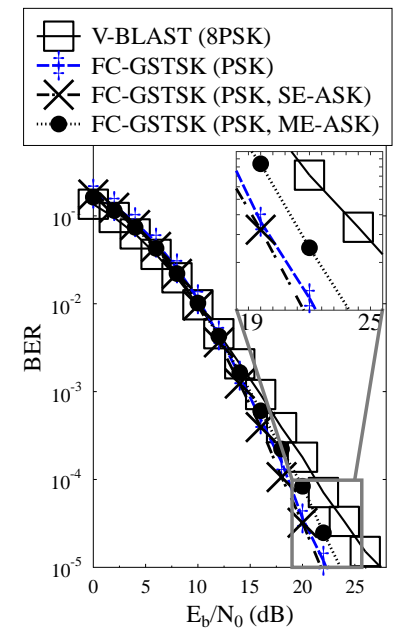

(a) $R=6.0, E_{b} / N_{0}$

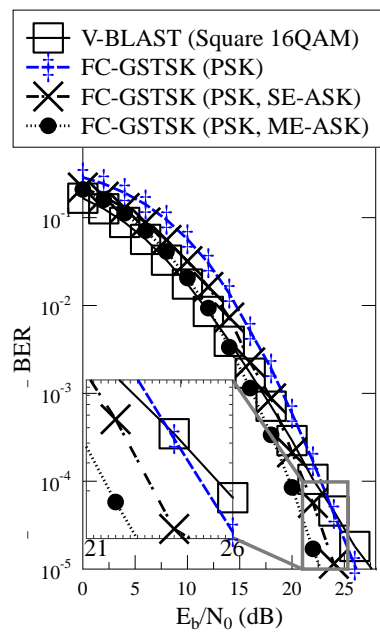

(c) $R=8.0, E_{b} / N_{0}$

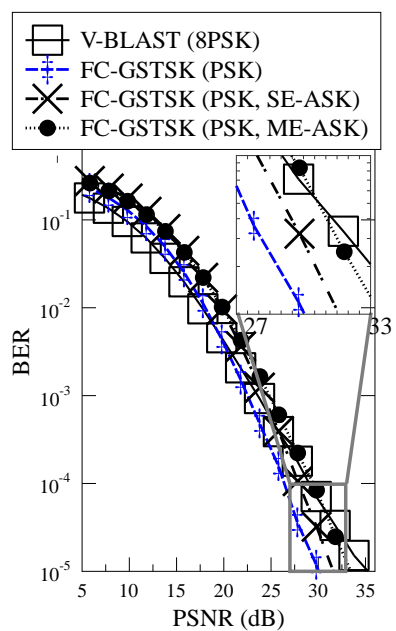

(b) $R=6.0$, PSNR

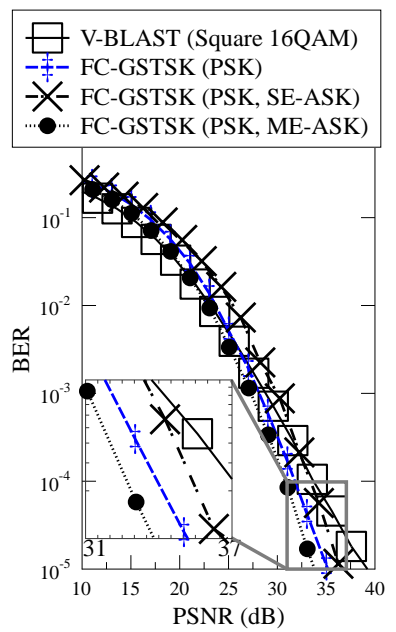

(d) $R=8.0$, PSNR
Fig. 7: Performance of FC-GSTSK using PSK and star QAM, when we have $M=T=2$ and $N=2$. The FC-GSTSK parameters are summarized in Table IV.

at high throughputs, the maximized diversity gain for a subset of signal matrices is sufficient to ensure a good performance.

Figs. 7(a) and 7(b) also showcase a tradeoff between bandwidth-efficiency and energy-efficiency, where the FCGSTSK(PSK,SE-ASK) using the star QAM signaling performs better than FC-GSTSK(PSK) in Fig. 7(a) owing to the improved bandwidth-efficiency of star QAM, but this trend is reversed in Fig. 7(b) owing to the increased PAPR of star QAM. Nonetheless, at a higher $R=8.0$, FCGSTSK(PSK,ME-ASK) is capable of outperforming both FCGSTSK(PSK) and FC-GSTSK(PSK,SE-ASK) with respect to both $E_{b} / N_{0}$ in Fig. 7(c) and PSNR in Fig. 7(d).

\section{B. Diversity-Rate (DR) Tradeoff}

The DR arrangement in [21], [62], [63] was originally conceived for the square-shaped signal matrix. In this work, however, we opt for using the rectangular-shaped signal matrix setup of $(T<M)$, so that the signal construction for large $M$ is based on the smaller $(T \times T)$ constituent matrices. More explicitly, for an integer of $(V=M / T)$, the DR arrangement models the $(T \times M)$ FC-GSTSK signal matrix as:

$$
\mathbf{S}=\sqrt{\frac{T}{M}}\left[\widetilde{\mathbf{S}}_{1} \widetilde{\mathbf{S}}_{2} \ldots \widetilde{\mathbf{S}}_{V}\right],
$$

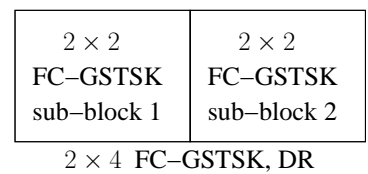

Fig. 8: Example of FC-GSTSK using the DR arrangement, when we have $(M=4),(T=2)$ and $(V=M / T=2)$.

where the $(T \times T)$ constituent matrices $\left\{\widetilde{\mathbf{S}}_{v}=\right.$ $\left.\sum_{\tau=1}^{T} s^{l_{\tau}} \widetilde{\mathbf{A}}_{\tau, \bar{q}_{\tau}}\right\}_{v=1}^{V}$ are all constructed by (18). The example of using $(M=4),(T=2)$ and $(V=M / T=2)$ is portrayed in Fig. 8. Furthermore, the LDC form of (40) is expressed as:

$$
\mathbf{S}=\sum_{v=1}^{V} \sum_{\tau=1}^{T} \sum_{\bar{q}=1}^{\bar{Q}} s_{v, \tau, \bar{q}} \mathbf{A}_{v, \tau, \bar{q}}=\sum_{q=1}^{Q} \bar{s}_{q} \overline{\mathbf{A}}_{q}
$$

where a total of $V T$ symbols are modulated by the $V$ number of $(T \times T)$ constituent FC-GSTSK:

$$
\bar{s}_{q}=s_{v, \tau, \bar{q}}=\left\{\begin{array}{ll}
s^{l_{v, \tau},}, & \text { for } \bar{q}=\bar{q}_{\tau} \\
0, & \text { for all the other } \bar{q}
\end{array},\right.
$$

while the dispersion matrix is given by:

$$
\overline{\mathbf{A}}_{q}=\mathbf{A}_{v, \tau, \bar{q}}=\left[\begin{array}{lllllll}
\mathbf{0} & \cdots & \mathbf{0} & \tilde{\mathbf{A}}_{\tau, \bar{q}_{\tau}}^{\tilde{T}_{\tau}} & \mathbf{0} & \cdots & \mathbf{0}
\end{array}\right]^{T} .
$$

The relationship between indices is given by $[q=\bar{q}+(\tau-$ $1) \bar{Q}+(v-1) \bar{Q} T]$. The total number of dispersion matrices in (41) is given by $(Q=V T \bar{Q})$. As a result, for the equivalent LDC received signal of (13), the $(1 \times Q)$-element vector $\overline{\mathbf{S}}$ is now given by (44). In summary, the transmit diversity order of the DR design is given by the reduced value of $[\min (M, T)=T=M / V]$, but the throughput is increased to $\left[R=V\left(\log _{2} \bar{Q}+\log L\right)\right]$.

\section{Reduced-RF Transmission}

The reduced-RF FC-GSTSK arrangement may be devised based on Remark 2 of Sec. III-A, which retains the full transmit diversity order of $M=T$. However, considering that the parameter optimization in Sec. III-C becomes increasingly challenging for a high $M$ at a higher $R$, we opt for invoking the alternative design of [51], where a total of $M_{A}=2$ out of $M$ TAs are activated for transmitting the $(2 \times 2)$ full-RF FC-GSTSK signal matrix over $T=2$ time slots. The resultant throughput is given by $\left[R=\left\lfloor\log _{2}\left(\begin{array}{c}M \\ M_{A}\end{array}\right)\right\rfloor+\log _{2} \bar{Q}+\log _{2} L\right]$. Specifically, the signal matrices for $M=4$ are given by [51]:

$$
\left.\mathbf{S}=\left\{\begin{array}{llll}
\widetilde{s}_{11} & \widetilde{s}_{12} & 0 & 0 \\
\widetilde{s}_{21} & \widetilde{s}_{22} & 0 & 0 \\
0 & \widetilde{s}_{11} & \widetilde{s}_{12} & 0 \\
0 & \widetilde{s}_{21} & \widetilde{s}_{22} & 0
\end{array}\right], \quad\left[\begin{array}{cccc}
0 & 0 & \widetilde{s}_{11} & \widetilde{s}_{12} \\
0 & 0 & \widetilde{s}_{21} & \widetilde{s}_{22} \\
0 & \widetilde{s}_{11} & 0 & \widetilde{s}_{12} \\
0 & \widetilde{s}_{21} & 0 & \widetilde{s}_{22}
\end{array}\right], e^{j \theta}\right\}
$$

where $\left\{\left\{\widetilde{s}_{a b}\right\}_{a=1}^{2}\right\}_{b=1}^{2}$ are signals taken from the $(2 \times 2)$ fullRF FC-GSTSK signal matrix $\widetilde{\mathbf{S}}$ constructed by (18), while the extra phase $\theta$ is used for maximizing the $\left(M_{A}=2\right)$-order diversity gain of (6).

\section{Performance Results}

Firstly, the diversity product $\Lambda_{p}$ and diversity sum $\Lambda_{s}$ results of (27) as well as the energy-efficiency metrics of PAPR and IAI are summarized in Sec. V-A. Secondly, our results recorded for $M=2$ TAs are presented in Sec. V-B, where the proposed FC-GSTSK is compared to the best-performing 


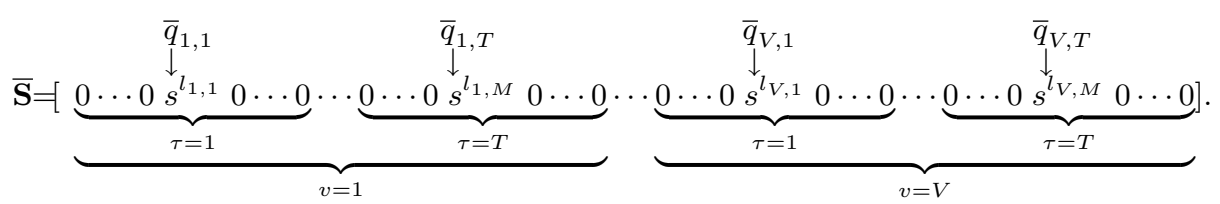

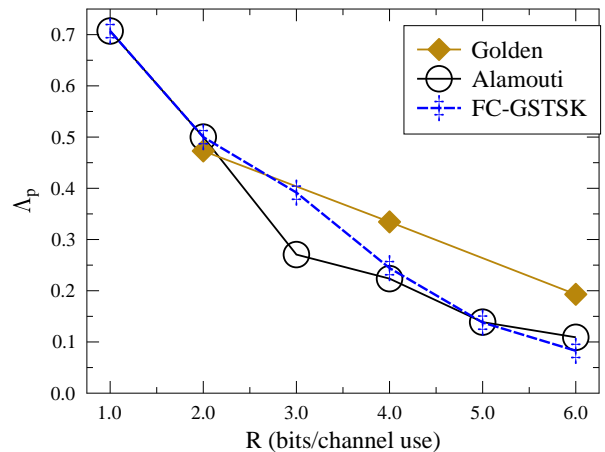

(a) Diversity Product $\Lambda_{p}$

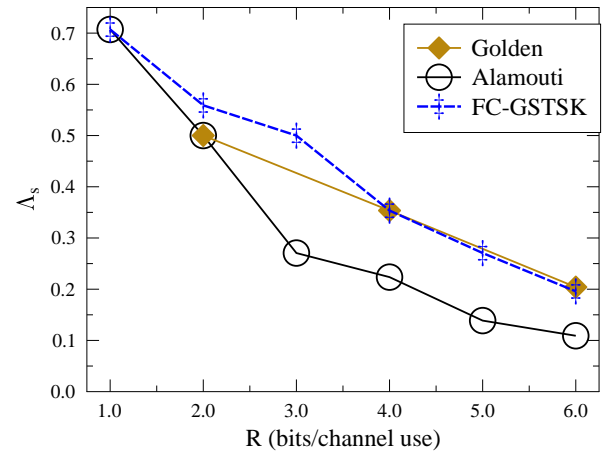

(b) Diversity Sum $\Lambda_{s}$

Fig. 9: Comparison of diversity product $\Lambda_{p}$ and diversity sum $\Lambda_{s}$ of (27), when we have $(M=T=2)$ and $(1 \leq R \leq 6)$. The FC-GSTSK parameters are summarized in Table V.

TABLE V: Parameters $\left(\bar{Q}, L, L_{D M}, L_{r}, \mathbf{u}\right)$ of FC-GSTSK (PSK) used in Fig. 9.

Fig. 9(a) $R=1.0:(1,2,2,4,[1,1]) \quad$ Fig. 9(a) $R=2.0:(2,2,4,4,[1,3])$

Fig. 9(a) $R=3.0:(4,2,8,14,[1,3]) \quad$ Fig. 9(a) $R=4.0:(8,2,16,14,[1,7])$

Fig. 9(a) $R=5.0:(16,2,32,3,[1,15])$ Fig. 9(a) $R=6.0:(8,8,64,19,[3,61])$

Fig. 9(b) $R=1.0:(1,2,2,4,[1,1]) \quad$ Fig. 9(b) $R=2.0:(4,1,5,8,[1,2])$

\begin{tabular}{l|l|l|}
\hline Fig. 9(b) $R=3.0:(2,4,16,8,[1,9])$ & Fig. 9(b) $R=4.0:(8,2,18,22,[1,11])$ \\
\hline Fig. 9(b) $R=5.0:(4,8,32,7,[1,9])$ & Fig. 9(b) $R=6.0:(16,4,68,45,11,9])$
\end{tabular}

\begin{tabular}{|l|l|}
\hline Fig. 9(b) $R=5.0:(4,8,32,7,[1,9])$ & Fig. 9(b) $R=6.0:(16,4,68,45,[1,9])$ \\
\hline
\end{tabular}

full-RF-based MIMO schemes. Thirdly, the performance advantages of FC-GSTSK are shown to be even more substantial for the increased number of $M=\{3,4\}$ TAs in Sec. V-B. Finally, our results of the channel coded schemes are offered in Sec. V-D. We note that since the most up-to-date $5 \mathrm{G}$ standard releases [82] currently support a maximum of four and eight transmission layers for the uplink and downlink scenarios, respectively, we opt for using up to $M=4$ TAs and up to $N=8$ RAs in this section.

\section{A. Characteristic Results}

As a full-diversity scheme, the proposed FC-GSTSK is compared to both the Golden code and to Alamouti's G2 STBC in terms of its diversity product $\Lambda_{p}$ and diversity sum $\Lambda_{s}$ of (27) in Fig. 9, where the associated FC-GSTSK parameters are summarized in Table V. On one hand, Fig. 9(a) demonstrates that the diversity product $\Lambda_{p}$ of FC-GSTSK becomes similar to Alamouti's G2 STBC as the throughput $R$ increases, while the Golden code achieves a higher $\Lambda_{p}$. On

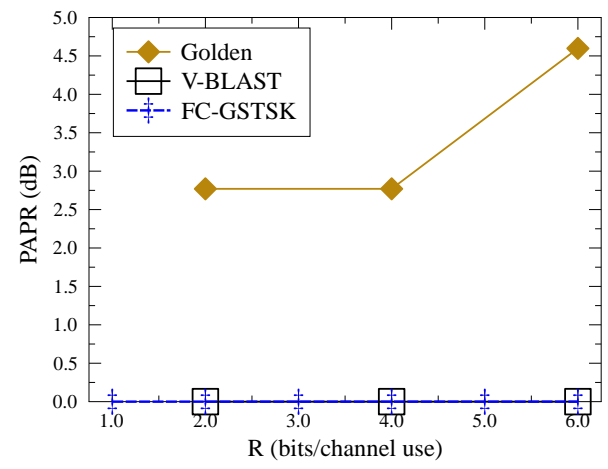

(a) PAPR, $R$

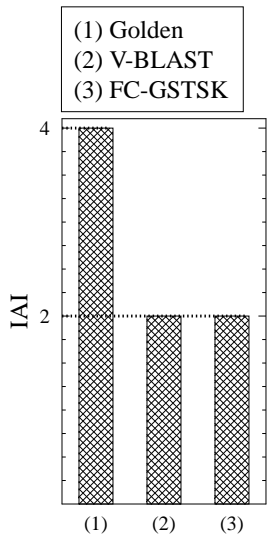

(c) IAI, $M=2$

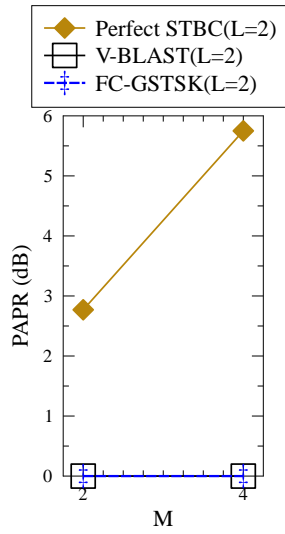

(b) PAPR, $M$

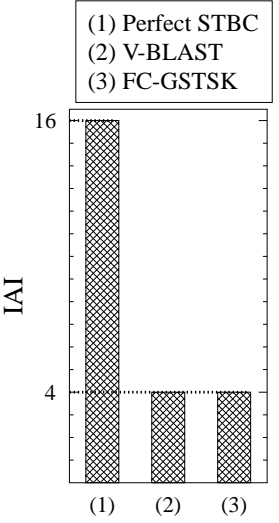

(d) IAI, $M=4$
Fig. 10: Comparison of the PAPR for signal transmission and the equivalent IAI for signal detection, when we have $(M=T=2)$ and $(1 \leq R \leq 6)$.

the other hand, Fig. 9(b) further evidences that the FC-GSTSK is capable of attaining superior diversity sums $\Lambda_{s}$, which are comparable to that of the Golden code at high throughputs. As discussed in Sec. III-C, the characteristics of Fig. 9 indicate that the FC-GSTSK may substantially outperform Alamouti's G2 STBC as $N$ increases.

As a high-rate scheme, the proposed FC-GSTSK exhibits the same level of transmission PAPR and IAI as V-BLAST, as demonstrated by Fig. 10. By contrast, despite their outstanding diversity gains, the perfect STBCs including the Golden code experience substantially increased transmission PAPR in Figs. 10(a) and 10(b). Moreover, the equivalent IAI $=M^{2}$ of perfect STBC is also shown to be a substantial extra price to pay compared to V-BLAST in Figs. 10(c) and 10(d).

\section{B. Performance Results for Two Transmit Antennas}

In Fig. 11, the BER performance versus the normalized average $E_{b} / N_{0}$ of FC-GSTSK is compared to the best performing MIMO schemes associated with $M=2$ TAs at throughputs of $(2 \leq R \leq 5)$. First of all, owing to the higher diversity sum $\Lambda_{s}$ seen in Fig. 9(b), FC-GSTSK starts to outperform Alamouti's G2 STBC as $N$ increases for the 


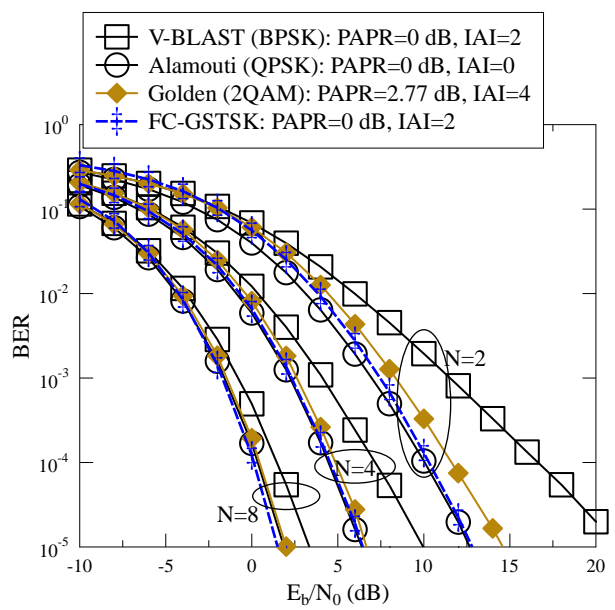

(a) $R=2.0, E_{b} / N_{0}$

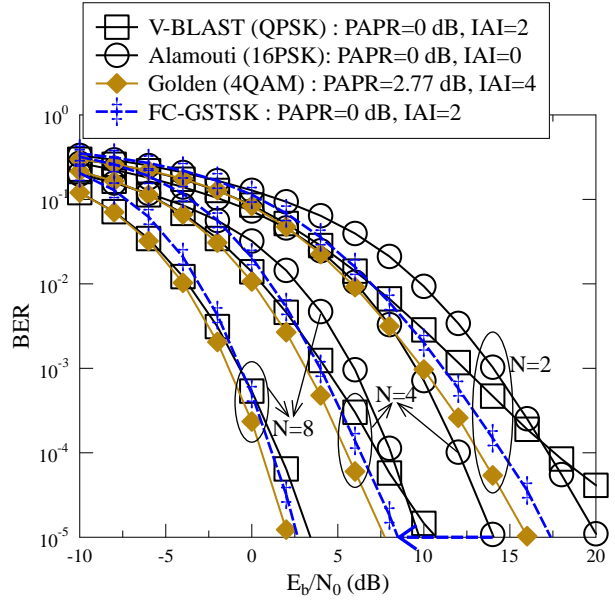

(c) $R=4.0, E_{b} / N_{0}$

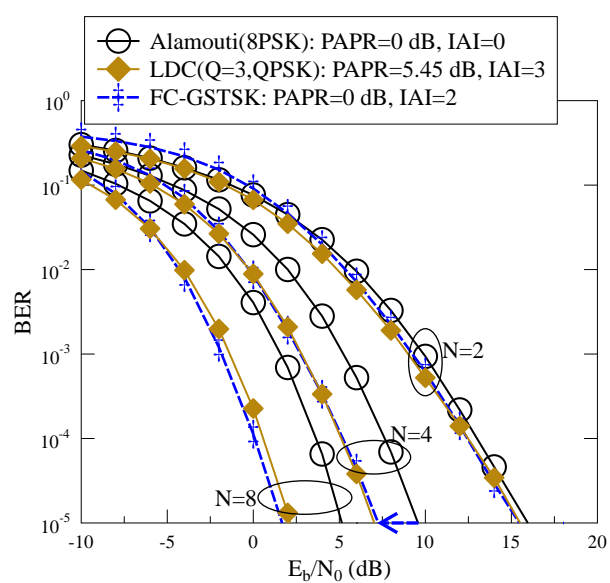

(b) $R=3.0, E_{b} / N_{0}$

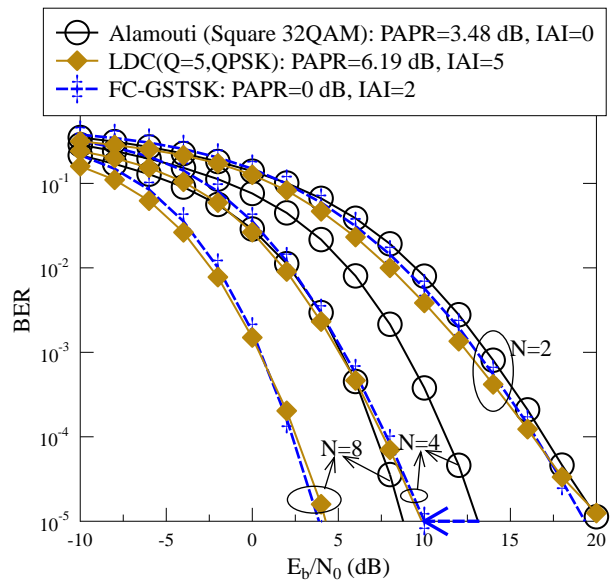

(d) $R=5.0, E_{b} / N_{0}$

Fig. 11: BER comparison (with respect to $E_{b} / N_{0}$ ) between the proposed FC-GSTSK, V-BLAST, Alamouti's G2 STBC [37], LDC [55], [74], [75], Golden code [36], when we have $(M=T=2)$ and $(N=\{2,4,8\})$. The FC-GSTSK parameters are summarized in Table VI.

TABLE VI: Parameters $\left(\bar{Q}, L, L_{D M}, L_{r}, \mathbf{u}\right)$ of FC-GSTSK (PSK) and parameters $\left(\bar{Q}, L, L_{D M}, L_{r}, \mathbf{u}, L_{A}, \alpha\right)$ of FC-GSTSK (PSK,ME-ASK) used in Figs. 11 and 12.

\begin{tabular}{|l|l|l|l|}
\hline & $N=2$ & $N=4$ & $N=8$ \\
\hline$R=2.0$ & PSK:(2,2,4,4,[1,3]) & PSK:(1,4,4,8,[1,1]) & PSK:(4,1,5,8,[1,2]) \\
\hline$R=3.0$ & PSK:(4,2,8,14,[1,3]) & PSK: $(2,4,16,8,[1,9])$ & PSK:(2,4,16,8,[1,9]) \\
\hline$R=4.0$ & PSK:(2,8,27,13,[1,19]) & PSK:(8,2,18,22,[1,11]) & PSK:(8,2,18,22,[1,11]) \\
\hline$R=5.0$ & PSK:(4,8,32,29,[1,15]) & PSK: $(4,8,32,7,[1,9])$ & PSK:(4,8,32,7,[1,9]) \\
\hline$R=6.0$ & PSK:(4,16,64,54,[7,57]) & PSK:(16,4,64,91,[1,45]) & PSK:(16,4,68,45,[1,9]) \\
\hline$R=8.0$ & ME-ASK:(4,16,64,23,[1,15],2,2.0) & ME-ASK: $(16,4,64,49,[1,37], 2,2.0)$ & ME-ASK: $(16,4,64,49,[1,37], 2,2.0)$ \\
\hline
\end{tabular}

case of $R=2.0$ in Fig. 11(a). More notably, Figs. 11(b)11(d) further evidence that FC-GSTSK massively outperforms Alamouti's G2 STBC for using $(N=\{2,4,8\})$ at $(R=$ $\{3.0,4.0,5.0\})$, as marked for $N=4$ in Figs. 11(b)-11(d). Secondly, observe in Figs. 11(a) and 11(c) that owing to the beneficial diversity gain, FC-GSTSK also exhibits a distinct performance improvement over V-BLAST for $(N=\{2,4,8\})$ at $(R=\{2.0,4.0\})$. However, the Golden code begins to excel in terms of its diversity gains in Fig. 11(c) as the throughput increases to $(R=4.0)$. Thirdly, Fig. 11(b) and 11(d) further demonstrate that FC-GSTSK associated with its perfect $0 \mathrm{~dB}$ PAPR is capable of achieving a comparable performance to that of the LDCs [55], [74], [75] using arbitrary signals. This verifies our energy-efficient FC-GSTSK signal construction, which achieves optimum performance without relying on arbitrary constellations.

In order to take into account the practical power consump- tion, the BER comparison (with respect to PSNR) between the proposed FC-GSTSK and the best-performing Golden code is further portrayed in Fig. 12. As expected, owing to its beneficial low-PAPR design, the proposed FC-GSTSK unequivocally outperforms the Golden code with respect to its PSNR, as demonstrated by Fig. 12.

\section{Performance Results for Three and Four Transmit Antennas}

For using $M=\{3,4\}$ TAs, Figs. 13(a) and 13(b) demonstrate that the FC-GSTSK consistently outperforms both VBLAST and STBCs. Furthermore, the FC-GSTSK is even capable of outperforming the perfect STBCs for $(M=N=$ $3, R=3.0)$ and $(M=N=4, R=4.0)$, as evidenced by Figs. 13(a) and 13(b), respectively. We note that owing to its substantially reduced PAPR, the advantage of FC-GSTSK over perfect STBC is further augmented versus the PSNR, which is not portrayed for Fig. 13 due to space limit. In summary, 


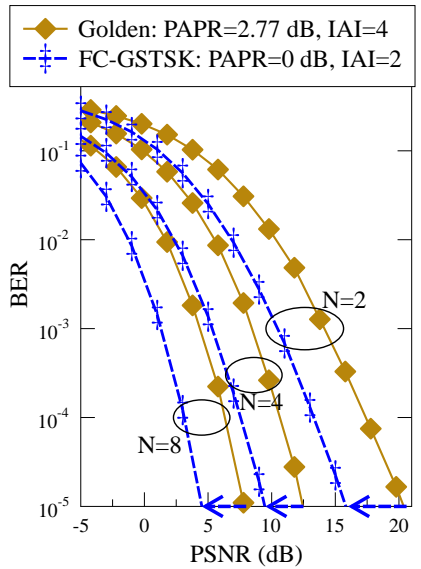

(a) $R=2.0$, PSNR

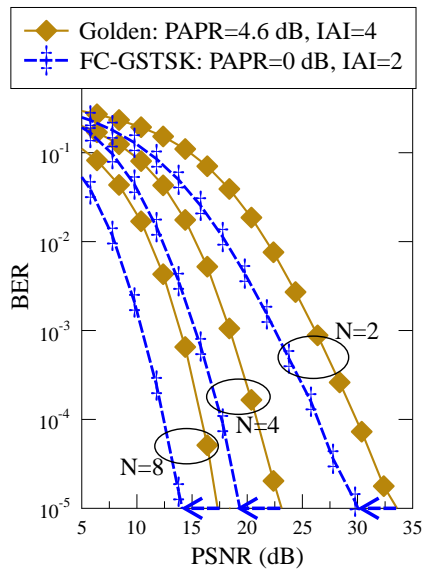

(c) $R=6.0$, PSNR

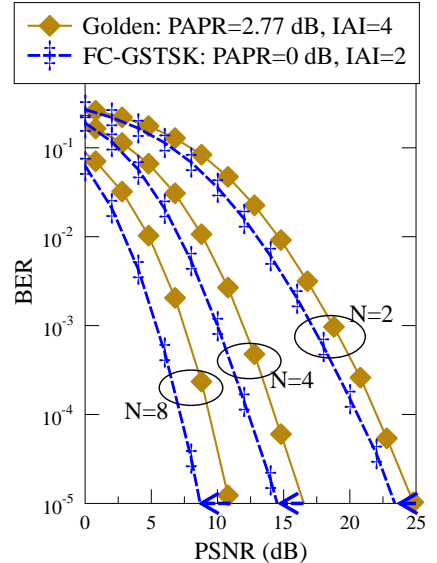

(b) $R=4.0$, PSNR

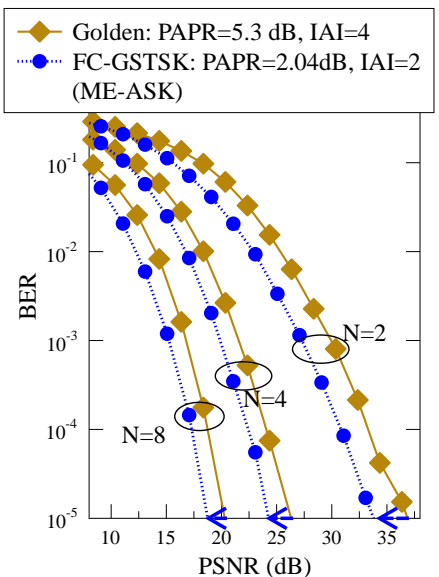

(d) $R=8.0$, PSNR

Fig. 12: BER comparison (with respect to PSNR) between the proposed FC-GSTSK and Golden code [36], when we have $(M=T=2)$, $(N=\{2,4,8\})$. The FC-GSTSK parameters are summarized in Table VI.

the impressive performance of FC-GSTSK is attributed to the diversity gain maximization proposed in Sec. III-C.

Moreover, for the case of $M=N=4$ at high throughputs of $R=\{6.0,8.0\}$, the DR arrangement of FC-GSTSK proposed in Sec. IV-B constructs the signal matrix for $M=4$ based on two constituent $(2 \times 2)$ FC-GSTSK signal matrices, which is shown to outperform both STBCs and V-BLAST in Figs. 13(c) and 13(d), respectively.

As a further advance, Fig. 14 demonstrates that when a reduced number of $\left(M_{A}=2\right)$ out of $(M=4)$ TAs are activated, the reduced-RF version of FC-GSTSK devised in Sec. IV-C also achieves an improved performance compared to its conventional counterparts of GSM [9] and STBC-SM [51] over a wide-range of throughputs of $(R=\{4.0,5.0,6.0,7.0\})$.

\section{Performance Results for Channel Coded Systems}

The Serially Concatenated Code (SCC) constituted by BCC and MIMO is portrayed in Fig. 15, where the iterative demapping and decoding is invoked at the two-stage turbo receiver. More explicitly, the source bits are passed through the BCC encoder and a interleaver before MIMO mapping at the SCC transmitter. At the turbo receiver, both the MIMO demodulator and BCC decoder accept and produce soft-bit decisions in the form of Log Likelihood Ratios (LLRs). The extrinsic LLRs $L_{M, e}$ produced by the MIMO demodulator are de-interleaved

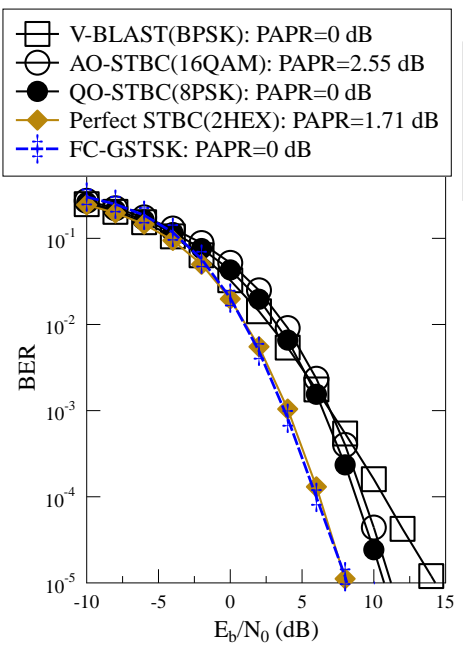

(a) $M=N=3, R=3.0$

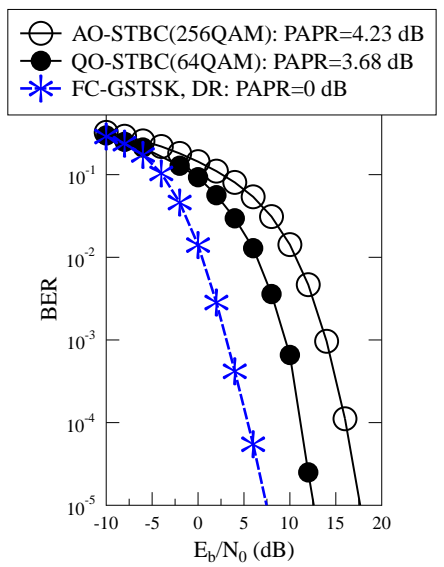

(c) $M=N=4, R=6.0$

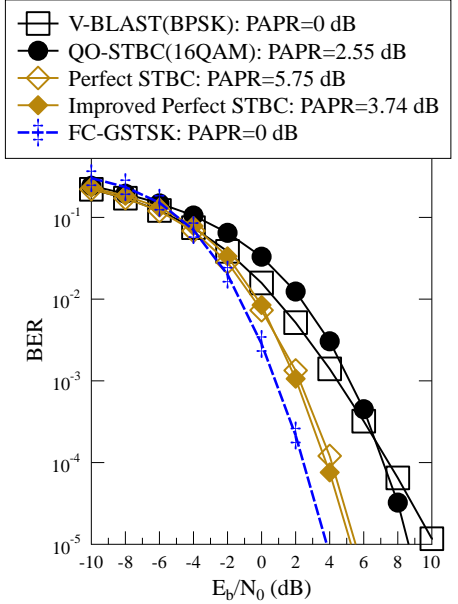

(b) $M=N=4, R=4.0$

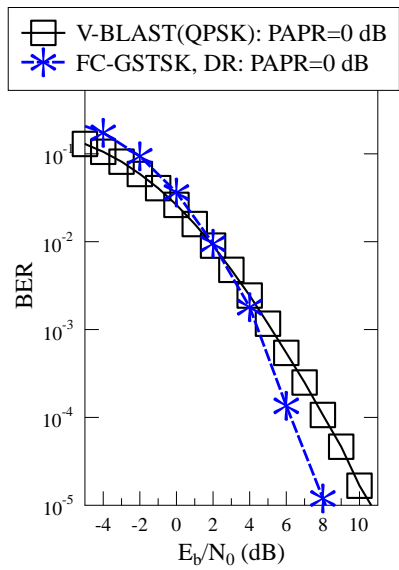

(d) $M=N=4, R=8.0$

Fig. 13: BER comparison (with respect to $E_{b} / N_{0}$ ) between the proposed FC-GSTSK, V-BLAST, AO-STBC [39], QO-STBC [42], perfect STBC [32], [33] and the improved perfect STBC [34]. The parameters $\left(\bar{Q}, L, L_{D M}, L_{r}, \mathbf{u}\right)$ of FC-GSTSK (PSK) associated with $(M=N=T=\{3,4\})$ and $(R=\{3.0,4.0\})$ in (a) and (b) are given by $(2,4,18,16,[1,7,13])$ and $(4,4,16,27,[1,5,9,13])$, respectively. The parameters $\left(\bar{Q}, L, L_{D M}, L_{r}, \mathbf{u}\right)$ of FC-GSTSK, DR (PSK) associated with $(M=N=T=4),(T=2, V=2)$ and $(R=\{6.0,8.0\})$ in (c) and (d) are given by $(2,4,16,8,[1,9])$ and $(8,2,18,22,[1,11])$, respectively.

and fed into the BCC encoder as the a priori LLRs $L_{D, a}$. Hence the extrinsic mutual information $\mathcal{I}_{M_{E}}=I\left(L_{M, e} ; b_{m}\right)$ at the output of the MIMO demodulator becomes equal to the a priori mutual information $\mathcal{I}_{D_{A}}=I\left(L_{D, a} ; b_{c}\right)$ at the input of the BCC decoder. Symmetrically, the extrinsic LLRs $L_{D, e}$ produced by the BCC decoder are interleaved and fed into the MIMO demodulator as the a priori LLRs $L_{M, a}$, which indicates that $\mathcal{I}_{D_{E}}=I\left(L_{D, e} ; b_{c}\right)$ becomes equal to $\mathcal{I}_{M_{A}}=I\left(L_{M, a} ; b_{m}\right)$ as seen in Fig. 15. The idealistic error-free detection may become achievable, when the full $\mathcal{I}_{D_{E}}=1.0$ is recovered by the BCC decoder.

According to the IEEE $802.11 \mathrm{a} / \mathrm{n} / \mathrm{ac} / \mathrm{ah}$ standard family, the BCC scheme invoked in this work is specified by the generator polynomials of $g_{0}=133_{8}$ and $g_{1}=171_{8}$ as well as the constrain length of $K=7$. The coding frame length is set to 10000 bits. The BCC rate is adjustable by puncturing, and we use $R_{c}=\{1 / 2,3 / 4,5 / 6\}$. In order to avoid ambiguity, the MIMO throughput is still represented by $R$, hence the overall coded system throughput is now given by $R_{c} R$. Moreover, without having to access the source bits at the transmitter, the turbo receiver may evaluate the mutual information in an 


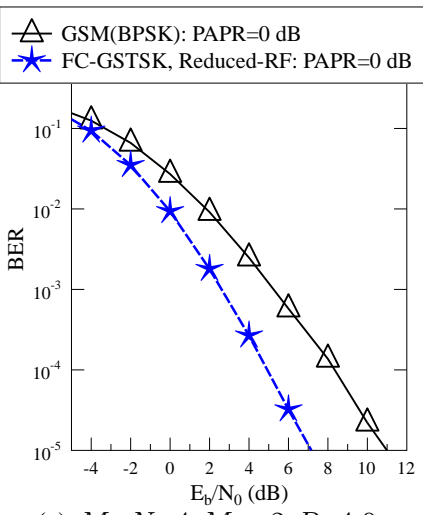

(a) $M=N=4, M_{A}=2, R=4.0$

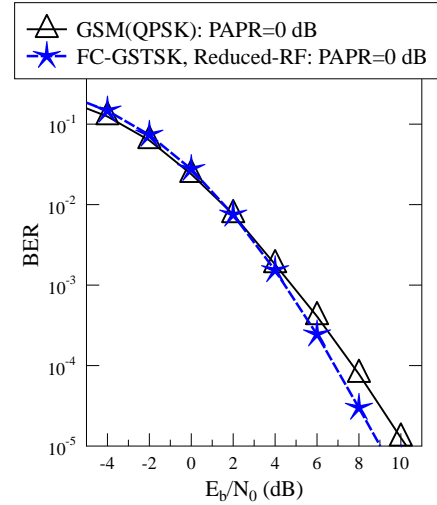

(c) $M=N=4, M_{A}=2, R=6.0$

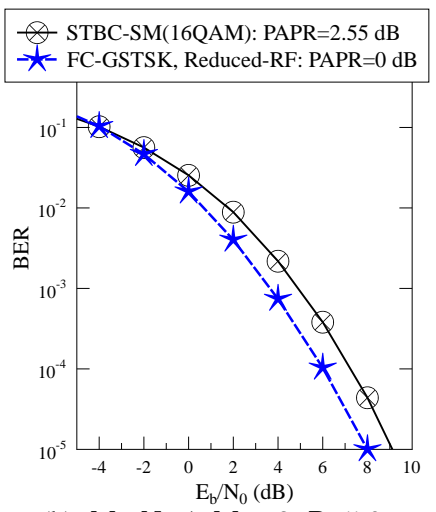

(b) $M=N=4, M_{A}=2, R=5.0$

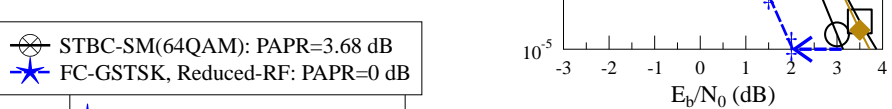

(a) $R_{c}=1 / 2, R=2.0,\left(E_{b} / N_{0}\right)$

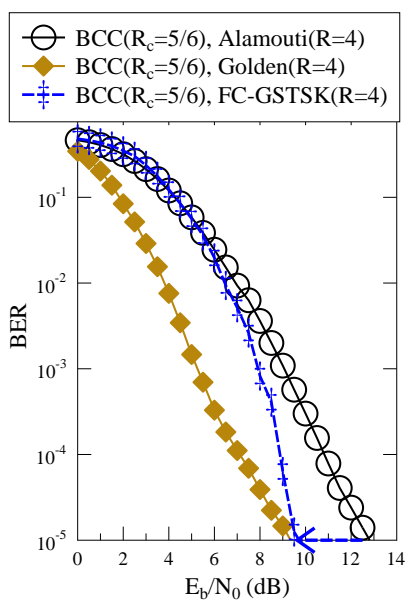

(c) $R_{c}=5 / 6, R=4.0,\left(E_{b} / N_{0}\right)$

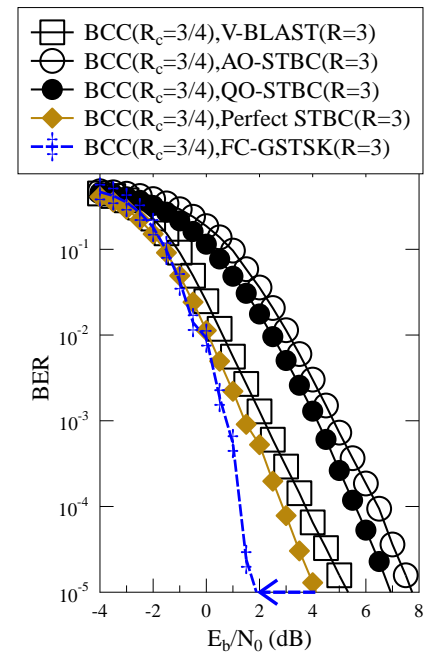

(b) $R_{c}=3 / 4, R=3.0,\left(E_{b} / N_{0}\right)$

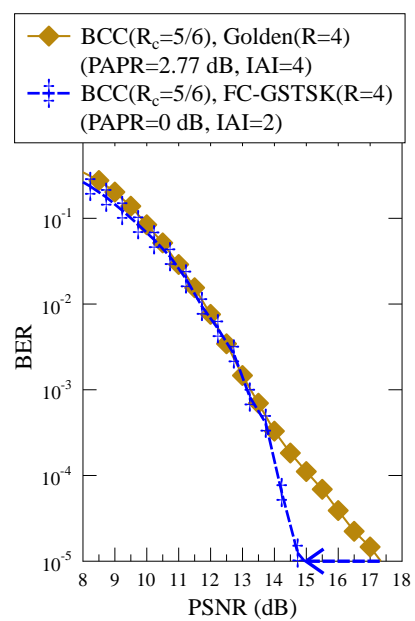

(d) $R_{c}=5 / 6, R=4.0,(P S N R)$

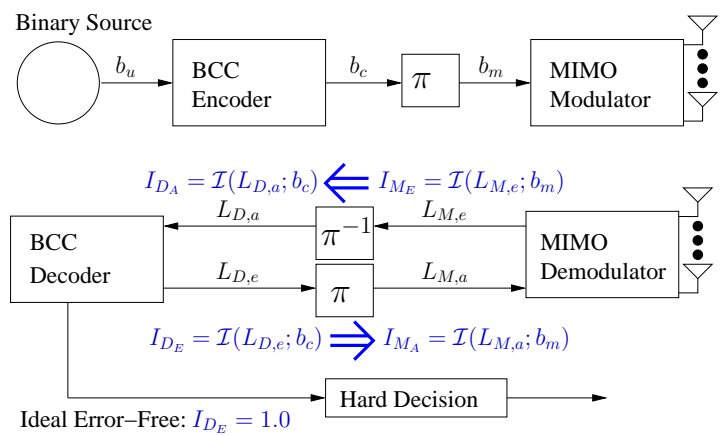

Fig. 15: Schematic of BCC coded MIMO scheme and its two-stage turbo decoder.

"on-line" fashion [52]-[55] as:

$\mathcal{I}(L ; b)=1+E\left[\frac{e^{L}}{1+e^{L}} \log _{2}\left(\frac{e^{L}}{1+e^{L}}\right)+\frac{1}{1+e^{L}} \log _{2}\left(\frac{1}{1+e^{L}}\right)\right]$,

so that the iterations may be terminated when no more information can be gleaned. In this work, we configure the maximum number of iterations between MIMO demapper and BCC decoder to a small number of 4 .

The convergence behaviour of the turbo receiver is visualized by the EXIT charts in Fig. 16. Notably, Fig. 16(a) evidences that compared to the conventioanl MIMO schemes of V-BLAST, STBC and Golden code, the proposed FC-
Fig. 17: BER comparison (with respect to $E_{b} / N_{0}$ and PSNR) between the proposed FC-GSTSK, V-BLAST, Alamouti's G2 STBC [37], AO-STBC [39], QO-STBC [42], Golden code [36] and perfect STBC [32], [33] in BCC coded systems. The parameters $\left(M, N, T, \bar{Q}, L, L_{D M}, L_{r}, \mathbf{u}\right)$ of FC-GSTSK associated with $R=\{2.0,3.0,4.0\}$ are given by $(2,2,2,2,2,4,4,[1,3])$, $(3,3,3,2,4,18,16,[1,7,13])$ and $(2,2,2,4,4,32,32,[1,19])$, respectively

GSTSK exhibits an enhanced iteration gain. This is due to the fact that instead of only mapping to the Gray coded PSK/QAM symbols, the FC-GSTSK maps the source bits to both the modulated symbol index and the dispersion matrix IM index, which offers additional degree of freedom. As a result, Fig. 16(b) demonstrates that the ideal error-free condition of $\mathcal{I}_{D_{E}}=1.0$ is achieved by the BCC FC-GSTSK scheme at $E_{b} / N_{0}=0 \mathrm{~dB}$, but its counterpart of Golden code can only obtain a lower $\mathcal{I}_{D_{E}}=0.98$, as shown in Fig. 16(c).

The EXIT charts analysis of Fig. 16 is further confirmed by the BER results of Fig. 17. First of all, Figs. 17(a) and 17(b) once again demonstrate that the proposed FC-GSTSK is capable of unequivocally outperforming the MIMO schemes of V-BLAST, Alamouti's G2 STBC, AO-STBC, QO-STBC, Golden code and perfect STBC in BCC coded systems. Secondly, although Goden code associated with the excessive IAI $=M^{2}$ appears to perform the best at a higher throughput seen in Fig. 17(c), the substantially increased PAPR of Golden 


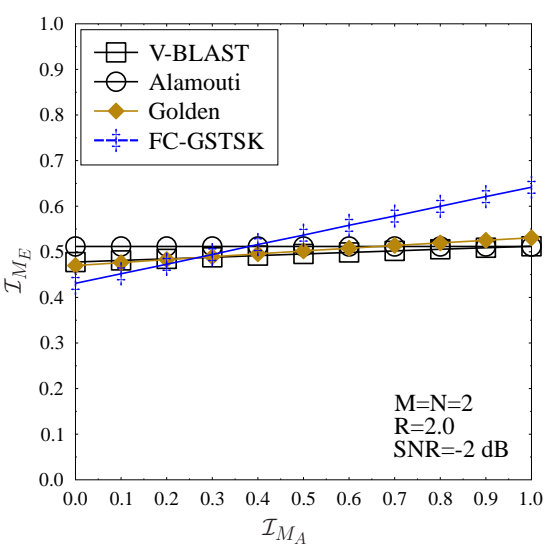

(a) EXIT charts

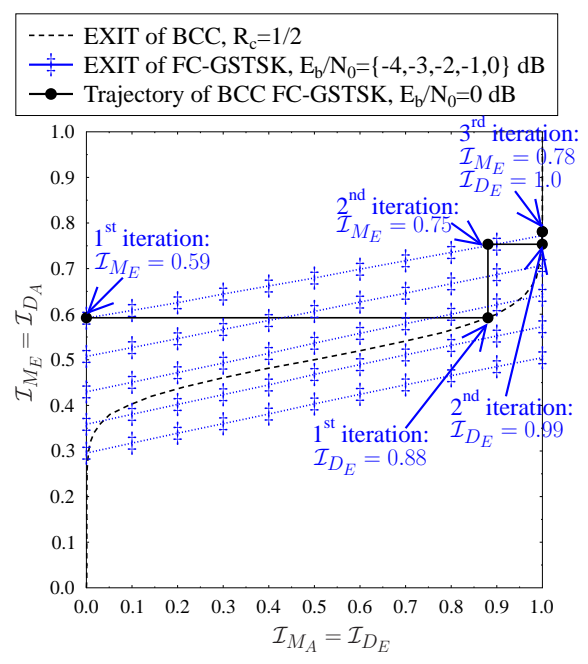

(b) BCC coded FC-GSTSK

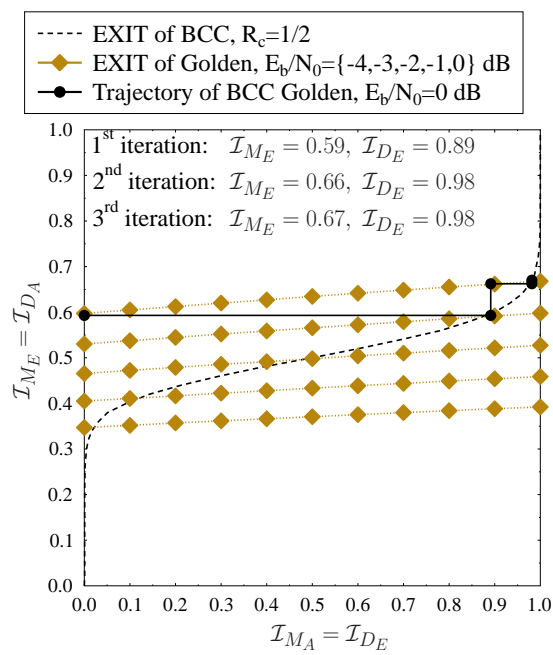

(c) BCC coded Golden code

Fig. 16: EXIT charts of the MIMO schemes $(M=N=2, R=2.0)$ and decoding trajectories of BCC coded FC-GSTSK and Golden code. The parameters are the same as those in Fig. 11(a).

code results in the evidently inferior performance compared to the proposed FC-GSTSK, when the PSNR takes into account the practical power consumption as shown in Fig. 17(d).

\section{CONCLUSIONS}

In this work, we have proposed a new FC-GSTSK scheme. It is unprecedented in the two decades of full-diversity highrate MIMO system design that as the FC-GSTSK outperforms both V-BLAST and STBC, the PAPR of signal transmission and the equivalent IAI of signal detection are reduced to the same level as that of V-BLAST. Moreover, the reduced-RF version of FC-GSTSK also outperforms GSM and STBC-SM without increasing the PAPR and the equivalent IAI. Owing to their explicit advantages in terms of energy-efficiency, bandwidth-efficiency and power-efficiency, the proposed FCGSTSK constitutes an attractive candidate for future wireless MIMO systems.

\section{DEDICATION}

Chao $\mathrm{Xu}$ would like to dedicate this paper to the fond memory of Sirui Hu (1985 - 2019), who was an outstanding scientist and an inspiration in the conception of this work.

\section{REFERENCES}

[1] J. G. Andrews, S. Buzzi, W. Choi, S. V. Hanly, A. Lozano, A. C. K. Soong, and J. C. Zhang, "What will 5G be?," IEEE J. Sel. Areas Commun., vol. 32, pp. 1065-1082, June 2014.

[2] S. Song, Y. Yang, Q. Xionq, K. Xie, B. Jeong, and B. Jiao, "A channel hopping technique I: theoretical studies on band efficiency and capacity," in Int. Conf. Commun., Circuits and Syst. (IEEE Cat.), vol. 1, pp. 229233 Vol.1, June 2004

[3] Y. A. Chau and S. Yu, "Space modulation on wireless fading channels," in IEEE 54th Veh. Technol. Conf. (VTC Fall 2001), vol. 3, pp. 1668-1671 vol.3, Oct 2001.

[4] R. Y. Mesleh, H. Haas, S. Sinanovic, C. W. Ahn, and S. Yun, "Spatial modulation," IEEE Trans. Veh. Technol., vol. 57, pp. 2228-2241, 2008.

[5] M. D. Renzo, H. Haas, A. Ghrayeb, S. Sugiura, and L. Hanzo, "Spatial modulation for generalized MIMO: Challenges, opportunities, and implementation," Proceedings of the IEEE, vol. 102, pp. 56-103, Jan 2014.

[6] P. Yang, Y. Xiao, Y. L. Guan, K. V. S. Hari, A. Chockalingam, S. Sugiura, H. Haas, M. D. Renzo, C. Masouros, Z. Liu, L. Xiao, S. Li, and L. Hanzo, "Single-carrier SM-MIMO: A promising design for broadband large-scale antenna systems," IEEE Commun. Surveys Tutorials, vol. 18, pp. 1687-1716, thirdquarter 2016.
[7] S. Sugiura, T. Ishihara, and M. Nakao, "State-of-the-art design of index modulation in the space, time, and frequency domains: Benefits and fundamental limitations," IEEE Access, vol. 5, pp. 21774-21790, 2017.

[8] E. Basar, M. Wen, R. Mesleh, M. D. Renzo, Y. Xiao, and H. Haas, "Index modulation techniques for next-generation wireless networks," IEEE Access, vol. 5, pp. 16693-16746, 2017.

[9] N. Ishikawa, S. Sugiura, and L. Hanzo, "50 years of permutation, spatial and index modulation: From classic RF to visible light communications and data storage," IEEE Commun. Surveys Tut., vol. 20, pp. 1905-1938, thirdquarter 2018.

[10] C. Xu, S. Sugiura, S. X. Ng, and L. Hanzo, "Spatial modulation and space-time shift keying: Optimal performance at a reduced detection complexity," IEEE Trans. Commun., vol. 61, pp. 206-216, January 2013.

[11] R. Rajashekar, K. V. S. Hari, and L. Hanzo, "Reduced-complexity ML detection and capacity-optimized training for spatial modulation systems," IEEE Trans. Commun., vol. 62, pp. 112-125, January 2014.

[12] P. K. Frenger and N. A. B. Svensson, "Parallel combinatory OFDM signaling," IEEE Trans. Commun., vol. 47, pp. 558-567, April 1999.

[13] E. Basar, "On multiple-input multiple-output OFDM with index modulation for next generation wireless networks," IEEE Trans. Signal Process., vol. 64, pp. 3868-3878, Aug 2016.

[14] N. Ishikawa, S. Sugiura, and L. Hanzo, "Subcarrier-index modulation aided OFDM - will it work?," IEEE Access, vol. 4, pp. 2580-2593, 2016.

[15] T. Ishihara and S. Sugiura, "Faster-Than-Nyquist signaling with index modulation," IEEE Wireless Commun. Lett., vol. 6, pp. 630-633, Oct 2017.

[16] T. Ishihara and S. Sugiura, "Iterative frequency-domain joint channel estimation and data detection of Faster-Than-Nyquist signaling," IEEE Trans. Wireless Commun., vol. 16, pp. 6221-6231, Sept 2017.

[17] T. Ishihara and S. Sugiura, "Differential Faster-Than-Nyquist signaling," IEEE Access, vol. 6, pp. 4199-4206, 2018.

[18] Y. Bian, M. Wen, X. Cheng, H. V. Poor, and B. Jiao, "A differential scheme for spatial modulation," in IEEE Global Commun. Conf., pp. 3925-3930, Dec 2013.

[19] Y. Bian, X. Cheng, M. Wen, L. Yang, H. V. Poor, and B. Jiao, "Differential spatial modulation," IEEE Trans. Veh. Technol., vol. 64, pp. 3262-3268, July 2015.

[20] C. Xu, R. Rajashekar, N. Ishikawa, S. Sugiura, and L. Hanzo, "Single$\mathrm{RF}$ index shift keying aided differential space-time block coding," IEEE Trans. Signal Process., vol. 66, pp. 773-788, Feb 2018.

[21] C. Xu, P. Zhang, R. Rajashekar, N. Ishikawa, S. Sugiura, L. Wang, and L. Hanzo, "Finite-cardinality single-RF differential space-time modulation for improving the diversity-throughput tradeoff," IEEE Trans. Commun. (accepted), 2018.

[22] E. Soujeri and G. Kaddoum, "The impact of antenna switching time on spatial modulation," IEEE Wireless Commun. Lett., vol. 5, pp. 256-259, June 2016.

[23] S. Sugiura, S. Chen, and L. Hanzo, "Coherent and differential spacetime shift keying: A dispersion matrix approach," IEEE Trans. Commun., vol. 58, pp. 3219-3230, November 2010.

[24] C. Xu, S. Sugiura, S. X. Ng, and L. Hanzo, "Reduced-complexity non- 
coherently detected differential space-time shift keying," IEEE Signal Process. Lett., vol. 18, pp. 153-156, March 2011.

[25] S. Sugiura, C. Xu, S. X. Ng, and L. Hanzo, "Reduced-complexity coherent versus non-coherent QAM-aided space-time shift keying," IEEE Trans. Commun., vol. 59, pp. 3090-3101, November 2011.

[26] S. Sugiura, S. Chen, and L. Hanzo, "Generalized space-time shift keying designed for flexible diversity-, multiplexing- and complexity-tradeoffs," IEEE Trans. Wireless Commun., vol. 10, pp. 1144-1153, April 2011.

[27] S. Sugiura, C. Xu, S. X. Ng, and L. Hanzo, "Reduced-complexity iterative-detection-aided generalized space-time shift keying," IEEE Trans. Veh. Technol., vol. 61, pp. 3656-3664, Oct 2012.

[28] B. S. Krongold and D. L. Jones, "PAR reduction in OFDM via active constellation extension," IEEE Trans. Broadcast., vol. 49, pp. 258-268, Sept 2003.

[29] Y. J. Kou, W. s. Lu, and A. Antoniou, "A new peak-to-average powerratio reduction algorithm for OFDM systems via constellation extension," IEEE Trans. Wireless Commun., vol. 6, pp. 1823-1832, May 2007.

[30] Y. Wang, W. Chen, and C. Tellambura, "Genetic algorithm based nearly optimal peak reduction tone set selection for adaptive amplitude clipping PAPR reduction," IEEE Trans. Broadcast., vol. 58, pp. 462-471, Sept 2012.

[31] S. H. Han and J. H. Lee, "An overview of peak-to-average power ratio reduction techniques for multicarrier transmission," IEEE Wireless Commun., vol. 12, pp. 56-65, April 2005.

[32] F. Oggier, G. Rekaya, J. C. Belfiore, and E. Viterbo, "Perfect spacetime block codes," IEEE Trans. Inf. Theory, vol. 52, pp. 3885-3902, Sept 2006.

[33] P. Elia, B. A. Sethuraman, and P. V. Kumar, "Perfect spacetime codes for any number of antennas," IEEE Trans. Inf. Theory, vol. 53, pp. 38533868, Nov 2007.

[34] K. P. Srinath and B. S. Rajan, "Improved perfect space-time block codes," IEEE Trans. Inf. Theory, vol. 59, pp. 7927-7935, Dec 2013.

[35] K. P. Srinath and B. S. Rajan, "Fast-decodable MIDO codes with large coding gain," IEEE Trans. Inf. Theory, vol. 60, pp. 992-1007, Feb 2014.

[36] J. C. Belfiore, G. Rekaya, and E. Viterbo, "The Golden code: a 2x2 full-rate space-time code with nonvanishing determinants," IEEE Trans. Inf. Theory, vol. 51, pp. 1432-1436, April 2005.

[37] S. M. Alamouti, "A simple transmit diversity technique for wireless communications," IEEE J. Sel. Areas Commun., vol. 16, pp. 1451-1458, Oct. 1998.

[38] V. Tarokh, H. Jafarkhani, and A. R. Calderbank, "Space-time block codes from orthogonal designs," IEEE Trans. Inf. Theory, vol. 45, pp. 1456-1467, July 1999.

[39] G. Ganesan and P. Stoica, "Space-time block codes: a maximum SNR approach," IEEE Trans. Inf. Theory, vol. 47, pp. 1650-1656, May 2001.

[40] G. Ganesan and P. Stoica, "Space-time diversity using orthogonal and amicable orthogonal designs," Wireless Pers. Commun., vol. 18, no. 2, pp. 165-178, 2001.

[41] H. Jafarkhani, "A quasi-orthogonal space-time block code," IEEE Trans. Commun., vol. 49, pp. 1-4, Jan 2001.

[42] W. Su and X. Xia, "Signal constellations for quasi-orthogonal spacetime block codes with full diversity," IEEE Trans. Inf. Theory, vol. 50, pp. 2331-2347, Oct 2004

[43] M. O. Sinnokrot and J. R. Barry, "Fast maximum-likelihood decoding of the Golden code," IEEE Trans. Wireless Commun., vol. 9, pp. 26-31, January 2010.

[44] J. Harshan and E. Viterbo, "Integer space-time block codes for practical MIMO systems," IEEE Wireless Commun. Lett., vol. 2, pp. 455-458, August 2013.

[45] T. Konishi, "Integer space-time block codes with large trace metrics," IEEE Wireless Commun. Lett., vol. 6, pp. 686-689, Oct 2017.

[46] E. Biglieri, Y. Hong, and E. Viterbo, "On fast-decodable space-time block codes," IEEE Trans. Inf. Theory, vol. 55, pp. 524-530, Feb 2009.

[47] C. Hollanti, J. Lahtonen, K. Ranto, R. Vehkalahti, and E. Viterbo, "On the algebraic structure of the Silver code: A 2 X2 perfect space-time block code," in IEEE Inf. Theory Workshop, pp. 91-94, May 2008.

[48] J. Jeganathan, A. Ghrayeb, and L. Szczecinski, "Generalized space shift keying modulation for MIMO channels," in IEEE 19th Int. Symp. Pers., Indoor Mobile Radio Commun., pp. 1-5, Sept 2008.

[49] J. Wang, S. Jia, and J. Song, "Generalised spatial modulation system with multiple active transmit antennas and low complexity detection scheme," IEEE Trans. Wireless Commun., vol. 11, pp. 1605-1615, April 2012.

[50] Y. Xiao, Z. Yang, L. Dan, P. Yang, L. Yin, and W. Xiang, "Lowcomplexity signal detection for generalized spatial modulation," IEEE Commun. Lett., vol. 18, pp. 403-406, March 2014.
[51] E. Basar, U. Aygolu, E. Panayirci, and H. V. Poor, "Space-time block coded spatial modulation," IEEE Trans. Commun., pp. 823-832, 2011.

[52] S. ten Brink, "Convergence of iterative decoding," Electron. Lett., vol. 35, pp. 806-808, May 1999.

[53] A. Ashikhmin, G. Kramer, and S. ten Brink, "Extrinsic information transfer functions: model and erasure channel properties," IEEE Trans. Inf. Theory, vol. 50, no. 11, pp. 2657-2673, 2004.

[54] L. Hanzo, O. Alamri, M. El-Hajjar, and N. Wu, Near-Capacity MultiFunctional MIMO Systems: Sphere-Packing, Iterative Detection and Cooperation. John Wiley \& Sons, May 2009.

[55] C. Xu, S. Sugiura, S. X. Ng, P. Zhang, L. Wang, and L. Hanzo, "Two decades of MIMO design tradeoffs and reduced-complexity MIMO detection in near-capacity systems," IEEE Access, vol. 5, pp. 18564 18632, 2017.

[56] P. A. Martin, "Differential spatial modulation for APSK in time-varying fading channels," IEEE Commun. Lett., vol. 19, pp. 1261-1264, 2015.

[57] J. Liu, L. Dan, P. Yang, L. Xiao, F. Yu, and Y. Xiao, "High-rate APSKaided differential spatial modulation: Design method and performance analysis," IEEE Commun. Lett., vol. PP, no. 99, pp. 1-1, 2016.

[58] B. L. Hughes, "Differential space-time modulation," IEEE Trans. Inf. Theory, vol. 46, pp. 2567-2578, Nov 2000.

[59] B. M. Hochwald and W. Sweldens, "Differential unitary space-time modulation," IEEE Trans. Commun., vol. 48, pp. 2041-2052, Dec 2000.

[60] B. L. Hughes, "Optimal space-time constellations from groups," IEEE Trans. Inf. Theory, vol. 49, pp. 401-410, Feb 2003.

[61] A. Shokrollahi, B. Hassibi, B. M. Hochwald, and W. Sweldens, "Representation theory for high-rate multiple-antenna code design," IEEE Trans. on Inf. Theory, vol. 47, pp. 2335-2367, Sep 2001.

[62] R. Rajashekar, N. Ishikawa, S. Sugiura, K. V. S. Hari, and L. Hanzo, "Full-diversity dispersion matrices from algebraic field extensions for differential spatial modulation," IEEE Trans. Veh. Technol., vol. 66, pp. 385-394, Jan 2017.

[63] R. Rajashekar, C. Xu, N. Ishikawa, S. Sugiura, K. V. S. Hari, an L. Hanzo, "Algebraic differential spatial modulation is capable of approaching the performance of its coherent counterpart," IEEE Trans. Commun., vol. 65, pp. 4260-4273, Oct 2017.

[64] X. Giraud, E. Boutillon, and J. C. Belfiore, "Algebraic tools to build modulation schemes for fading channels," IEEE Trans. Inf. Theory, vol. 43, pp. 938-952, May 1997.

[65] V. M. DaSilva and E. S. Sousa, "Fading-resistant modulation using several transmitter antennas," IEEE Trans. Commun., pp. 1236-1244, 1997.

[66] J. Boutros and E. Viterbo, "Signal space diversity: a power- and bandwidth-efficient diversity technique for the Rayleigh fading channel," IEEE Trans. Inf. Theory, vol. 44, pp. 1453-1467, Jul 1998.

[67] H. E. Gamal and M. O. Damen, "Universal space-time coding," IEEE Trans. Inf. Theory, vol. 49, pp. 1097-1119, May 2003.

[68] M. O. Damen, K. Abed-Meraim, and J. C. Belfiore, "Diagonal algebraic space-time block codes," IEEE Trans. Inf. Theory, pp. 628-636, 2002.

[69] P. Elia, K. R. Kumar, S. A. Pawar, P. V. Kumar, and H. F. Lu, "Explicit space-time codes achieving the diversity-multiplexing gain tradeoff," IEEE Tran. Inf. Theory, vol. 52, pp. 3869-3884, Sept 2006.

[70] L. Zheng and D. N. C. Tse, "Diversity and multiplexing: a fundamental tradeoff in multiple-antenna channels," IEEE Trans. Inf. Theory, vol. 49, pp. 1073-1096, May 2003.

[71] B. A. Sethuraman, B. S. Rajan, and V. Shashidhar, "Full-diversity, highrate space-time block codes from division algebras," IEEE Trans. Inf. Theory, vol. 49, pp. 2596-2616, Oct 2003.

[72] K. T and B. S. Rajan, "STBC-schemes with nonvanishing determinant for certain number of transmit antennas," IEEE Trans. Inf. Theory, vol. 51, pp. 2984-2992, Aug 2005.

[73] J. Harshan and E. Viterbo, "On the robustness of algebraic stbcs to coefficient quantization," in 2012 Australian Commun. Theory Workshop (AusCTW), pp. 55-60, Jan 2012.

[74] R. W. Heath and A. J. Paulraj, "Linear dispersion codes for MIMO systems based on frame theory," IEEE Trans. Signal Process., vol. 50, pp. 2429-2441, Oct 2002.

[75] L. Hanzo, O. Alamri, M. El-Hajjar, and N. Wu, Near-Capacity MultiFunctional MIMO Systems: Sphere-Packing, Iterative Detection and Cooperation. Wiley-IEEE Press, 2009.

[76] X. Liang and X. Xia, "Unitary signal constellations for differential space-time modulation with two transmit antennas: parametric codes, optimal designs, and bounds," IEEE Trans. Inf. Theory, vol. 48, pp. 22912322, Aug 2002.

[77] B. Hassibi and B. M. Hochwald, "High-rate codes that are linear in space and time," IEEE Trans. Inf. Theory, pp. 1804-1824, 2002. 
[78] T. May, H. Rohling, and V. Engels, "Performance analysis of Viterbi decoding for 64-DAPSK and 64-QAM modulated OFDM signals," IEEE Trans. Commun., vol. 46, pp. 182-190, Feb. 1998.

[79] X. Dong, N. Beaulieu, and P. Wittke, "Error probabilities of twodimensional $M$-ary signaling in fading," IEEE Trans. Commun., vol. 47, pp. 352-355, Mar. 1999.

[80] C. Xu, S. X. Ng, and L. Hanzo, "Multiple-symbol differential sphere detection and decision-feedback differential detection conceived for differential qam," IEEE Trans. Veh. Technol., vol. 65, pp. 8345-8360, Oct 2016.

[81] C. Xu, L. Wang, S. X. Ng, and L. Hanzo, "Soft-decision multiplesymbol differential sphere detection and decision-feedback differential detection for differential qam dispensing with channel estimation in the face of rapidly fading channels," IEEE Trans. Wireless Commun., vol. 15, pp. 4408-4425, June 2016.

[82] 3GPP Technical Report 21.915, "Summary of rel-15 work items," [Online]. Available: http://www.3gpp.org/release-15.

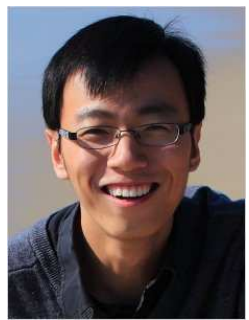

Chao Xu (S'09-M'14) received a B.Eng. degree from Beijing University of Posts and Telecommunications, China, and a BSc(Eng) with First Class Honours from Queen Mary, University of London, UK, through a Sino-UK joint degree program in 2008. He obtained a MSc degree with distinction in Radio Frequency Communication Systems and a Ph.D. degree in Wireless Communications from the University of Southampton, UK in 2009 and 2015, respectively. He is currently a postdoctoral researcher working at Next Generation Wireless Research Group, University of Southampton, UK. His research interests include index modulation, reduced-complexity MIMO design, full-duplex, noncoherent detection and turbo detection. He was awarded the Best M.Sc. Student in Broadband and Mobile Communication Networks by the IEEE Communications Society (United Kingdom and Republic of Ireland Chapter) in 2009. He also received 2012 Chinese Government Award for Outstanding Self-Financed Student Abroad.

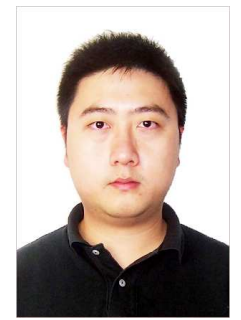

Peichang Zhang received the B.Eng. degree (Hons.) in electronic engineering from the University of Central Lancashire, Preston, U.K., in 2009 and the M.Sc. and Ph.D degree in wireless communications from the University of Southampton, Southampton, U.K., in 2010 and 2015, respectively. He is now with the College of Information and Engineering, Shenzhen University, China. His research interests include antenna selection, coherent and non-coherent detection, and channel estimation, internet of things.

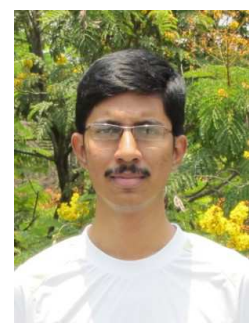

Rakshith Rajashekar (M'14-SM'17) received the B.E. degree in electrical communication engineeringfrom Visvesvaraya Technological University, Kar-nataka, India, in 2007. He received his Ph.D. fromthe Department of Electrical Communication Engineering, Indian Institute of Science (IISc), India, in 2014. He was a Research Fellow at the University of Southampton, UK from 2015 to 2019. Before joining the University of Southampton, he worked at Accord Software \& Systems, Bengaluru, India, as a Systems Engineer from 2007 to 2009, and as a Senior Scientist at Broadcom Communications, Bengaluru, India from 2014 to 2015. His research interests include antenna selection in MIMO systems, differential communication, millimeter wave communication, communication between drones with a focus on space-time signal processing and coding. $\mathrm{He}$ is the recipient of the Special Recognition award from Broadcom Communications, India and the Deans Award from the University of Southampton, UK, for excellence in research. He has received the Best Reviewer award from IEEE Transactions on Wireless Communications, IEEE Transactions on Communications and IEEE Wireless Communications Letters.

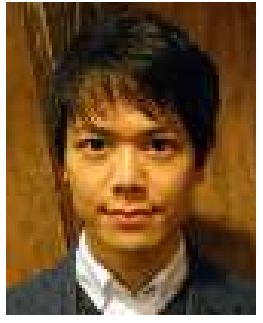

Naoki Ishikawa (S'13-M'17) was born in Kanagawa, Japan, in 1991. He received the B.E., M.E., and $\mathrm{Ph} . \mathrm{D}$. degrees from the Tokyo University of Agriculture and Technology, Tokyo, Japan, in 2014, 2015, and 2017, respectively. From June 2015 to September 2015, he was an academic visitor with the School of Electronics and Computer Science, University of Southampton, UK. From April 2016 to March 2017, he was a research fellow of the Japan Society for the Promotion of Science. From April 2017, he has been an assistant professor in the Graduate School of Information Sciences, Hiroshima City University, Japan. He was certified as an Exemplary Reviewer of IEEE TRANSACTIONS ON COMMUNICATIONS 2017. He also received nine domestic awards, including the Yasujiro Niwa Outstanding Paper Award from Tokyo Denki University in 2018, the Telecom System Technology student Award (honorable mention) from Telecommunications Advancement Foundation of Japan in 2014, and the Outstanding Paper Award for Young C\&C Researchers from NEC C\&C Foundation in 2014.

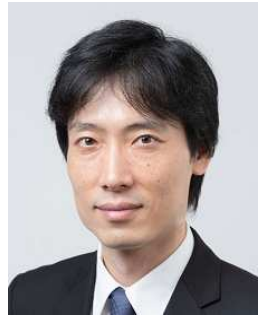

Shinya Sugiura (M'06-SM'12) received the B.S. and M.S. degrees in aeronautics and astronautics from Kyoto University, Kyoto, Japan, in 2002 and 2004 , respectively, and the Ph.D. degree in electronics and electrical engineering from the University of Southampton, Southampton, U.K., in 2010.

From 2004 to 2012, he was a Research Scientist with Toyota Central Research and Development Laboratories, Inc., Aichi, Japan. From 2013 to 2018, he was an Associate Professor with the Department of Computer and Information Sciences, Tokyo University of Agriculture and Technology, Tokyo, Japan. Since 2018, he has been an Associate Professor with the Institute of Industrial Science, The University of Tokyo, Tokyo, Japan, where he heads the Wireless Communications Research Group. His research has covered a range of areas in wireless communications, networking, signal processing, and antenna technology. $\mathrm{He}$ authored or coauthored over 70 IEEE journal papers.

Dr. Sugiura was a recipient of a number of awards, including the Fifth Yasuharu Suematsu Award in 2019, the Sixth RIEC Award from the Foundation for the Promotion of Electrical Communication in 2016, the Young Scientists' Prize by the Minister of Education, Culture, Sports, Science and Technology of Japan in 2016, the 14th Funai Information Technology Award (First Prize) from the Funai Foundation in 2015, the 28th Telecom System Technology Award from the Telecommunications Advancement Foundation in 2013, the Sixth IEEE Communications Society Asia-Pacific Outstanding Young Researcher Award in 2011, the 13th Ericsson Young Scientist Award in 2011, and the 2008 IEEE Antennas and Propagation Society Japan Chapter Young Engineer Award. He was also certified as an Exemplary Reviewer of IEEE COMmUniCATIONS LETTERS in 2013 and 2014, and that of IEEE TRANSACTIONS ON COMMUNICATIONS in 2018. 


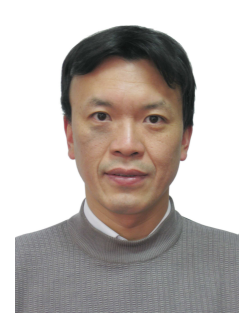

Zhaocheng Wang Prof. Zhaocheng Wang received his B.S., M.S. and Ph.D. degrees from Tsinghua University in 1991, 1993 and 1996, respectively. From 1996 to 1997, he was a Post-Doctoral Fellow with Nanyang Technological University, Singapore. From 1997 to 1999, he was a Research Engineer/Senior Engineer with OKI Techno Centre Pte. Ltd., Singapore. From 1999 to 2009, he was a Senior Engineer/Principal Engineer with Sony Deutschland GmbH, Germany. Since 2009, he has been a Professor with Department of Electronic Engineering, Tsinghua University, where he is currently the Director of Broadband Communication Key Laboratory, Beijing National Research Center for Information Science and Technology (BNRist).

His research interests include wireless communications, millimeter wave communications and optical wireless communications. Prof. Wang published more than 150 peer-reviewed journal papers. He hold 34 US/EU granted patents ( 23 of them as the first inventor). Several granted US/EU patents have been accepted by a plurality of international standards like IEEE802.11n, DVB-T2, IEEE802.15.3c, ECMA387 and etc. Besides that, he authored or co-authored two books, which have been selected by IEEE Series on Digital \& Mobile Communication and published by Wiley-IEEE Press.

Prof. Wang is a Fellow of the Institution of Engineering and Technology. He received ICC2013 Best Paper Award, OECC2015 Best Student Paper Award, 2016 IEEE Scott Helt Memorial Award (Best Paper Award of IEEE Transactions on Broadcasting), 2016 IET Premium Award (Best Paper Award of Electronics Letters), 2016 National Award for Science and Technology Progress (First Prize), ICC2017 Best Paper Award and 2018 IEEE ComSoc Asia-Pacific Outstanding Paper Award. He was an Associate Editor of IEEE Transactions on Wireless Communications from 2011 to 2015 and an Associate Editor of IEEE Communications Letters from 2013 to 2016. He was also Signal Processing for Optical Wireless Communications Symposium CoChair of IEEE GlobalSIP2015, Optical Wireless Communications Symposium Co-Chair of OECC2015 and Wireless Communications Symposium Co-Chair of IEEE ICC 2013.

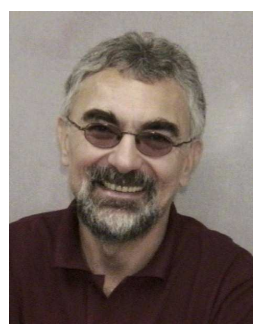

Lajos Hanzo (M'91-SM'92-F'04) Lajos Hanzo (http://www-mobile.ecs.soton.ac.uk) FREng, F'04, FIET, Fellow of EURASIP, received his 5-year degree in electronics in 1976 and his doctorate in 1983 from the Technical University of Budapest. In 2009 he was awarded an honorary doctorate by the Technical University of Budapest and in 2015 by the University of Edinburgh. In 2016 he was admitted to the Hungarian Academy of Science. During his 40-year career in telecommunications he has held various research and academic posts in Hungary, Germany and the UK. Since 1986 he has been with the School of Electronics and Computer Science, University of Southampton, UK, where he holds the chair in telecommunications. He has successfully supervised $119 \mathrm{PhD}$ students, co-authored 18 John Wiley/IEEE Press books on mobile radio communications totalling in excess of 10000 pages, published $1800+$ research contributions at IEEE Xplore, acted both as TPC and General Chair of IEEE conferences, presented keynote lectures and has been awarded a number of distinctions. Currently he is directing a 60 -strong academic research team, working on a range of research projects in the field of wireless multimedia communications sponsored by industry, the Engineering and Physical Sciences Research Council (EPSRC) UK, the European Research Council's Advanced Fellow Grant and the Royal Society's Wolfson Research Merit Award. He is an enthusiastic supporter of industrial and academic liaison and he offers a range of industrial courses. He is also a Governor of the IEEE ComSoc and VTS. He is a former Editor-in-Chief of the IEEE Press and a former Chaired Professor also at Tsinghua University, Beijing. For further information on research in progress and associated publications please refer to http://www-mobile.ecs.soton.ac.uk 